\title{
Chirality in Conjugated Polymers: When Two Components Meet.
}

\author{
Michiel Verswyvel ${ }^{* a}$ and Guy Koeckelberghs ${ }^{a}$. \\ Received (in $X X X, X X X)$ Xth $X X X X X X X X X 20 X X$, Accepted $X$ th $X X X X X X X X X 20 X X$ \\ DOI: $10.1039 / \mathrm{b} 000000 \mathrm{x}$
}

\begin{abstract}
${ }_{5}$ Chirality is a well-studied feature in the auspicious class of conjugated polymers. Proper use allows to study and to control the behavior of the polymers which make them very valuable in many applications. Mostly chiral homopolymers are considered, but what happens when the chirality originates from the interplay between multiple components? This review summarizes different approaches for multicomponent chirality in conjugated polymers and properties of them.
\end{abstract}

\section{Introduction}

Conjugated polymers $(\mathrm{CP})$ received a lot of attention since their discovery a few decades ago. This continued interest mainly originates from the fact that they show unique properties which 15 allow them to be useful in many applications. Indeed, while originally recognized as plastics that could be electrically conductive, they are nowadays used in oLED, oFETs and solar cells, amongst others. Their unique properties originate to a large extent from their supra- and/or macromolecular structure.

${ }_{20}$ Consequently, chirality, an aspect related to symmetry, can add additional features to $\mathrm{CP}$, as it can give rise to structures, and, related with them properties, that are absent in the achiral analogues. As such, chirality enables more techniques to probe the supra- and macromolecular structure of the materials, such as 25 circular dichroism (CD) and circular polarized luminescence (CPL) and it can give access to the field of enantioselective synthesis, via chiral catalysts or chiral chromatography, and enantioselective sensing. As conjugated polymers typically contain solubilizing alkyl groups, the most straightforward way to 30 introduce chirality is to attach chiral substituents to generate chiral homopolymers. In this way, a plethora of conjugated polymers, with different backbones and substituents, has been prepared and their (chiral) properties have been investigated. Alternatively, chiral $\mathrm{A}_{2}$-functionalized monomers can be reacted 35 with achiral $\mathrm{B}_{2}$-functionalized monomers in a polycondensation reaction, resulting in alternating copolymers. However, according

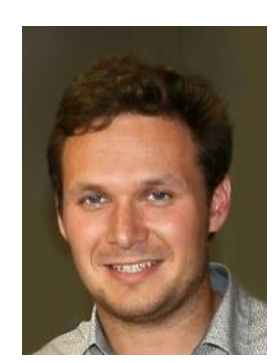

Michiel Verswyvel is a $3^{\text {rd }}$ year PhDstudent, fellow of FWO-Vlaanderen, affiliated with the Laboratory of Polymer Synthesis at the University of Leuven in Belgium. At the same university, he received his Bachelor (2007) and Master (2009) degree. His research interests include the synthesis and characterization of conjugated Michiel Verswyvel block polymers. to their molecular structure, they can be regarded as homopolymers, comprised of an $\mathrm{AB}$ repeating unit. As a consequence, both approaches result in 'one component systems'. ${ }_{40}$ These polymers have been reviewed at different occasions and they are not the subject of our review. ${ }^{1}$

A second way to combine chirality and conjugated polymers is to chirally align well defined chiral oligomers by chiral moieties, such as chiral binaphthol ${ }^{2,3}$ and cyclohexanediamine ${ }^{4}$.

${ }_{45}$ Apart from homopolymers, research on CP in general is more and more focused on multicomponent systems, such as block copolymers, random copolymers, (inorganic) nanoparticles decorated with CP, surfaces covered with CPs, etc. These materials combine the properties of the constituting components 50 into one material, giving rise to properties that are not accessible in 'one component' materials. Moreover, the presence of one component can influence the macro- and supramolecular organization of the second component, giving rise to a structure and inferred properties of that second component that cannot be 55 obtained in the homopolymer.

Interestingly, chirality can also be incorporated in multicomponent systems, in which one or more components are chiral. The presence of different component(s) can influence the macro- and supramolecular structure, giving rise to 60 unprecedented chiral behavior. In this review chiral conjugated polymers in 'multicomponent systems' will be discussed. More in particular, we will deal with conjugated random copolymers, conjugated block copolymers and (achiral) conjugated polymers in the presence of chiral additive or media.

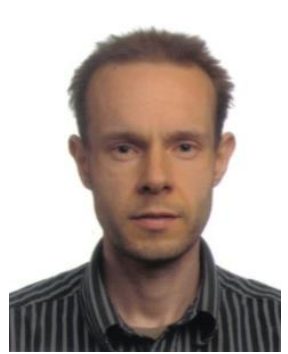

Guy Koeckelberghs is assistant professor at the $K U$ Leuven. He received his $P h D$ in 2002 at $K U$ Leuven on the synthesis of chiral polymers for nonlinear optical applications in the group of Prof. $C$. Samyn. His research interests are the synthesis and chiroptical properties of allconjugated block copolymers and the magnetic and nonlinear optical properties Guy Koeckelberghs of conjugated polymers. 
।

\section{Figure 1. Bisignate Cotton effects originating from chiral exciton coupling. Chiral organization and expression}

\section{${ }_{5}$ Chiral organization}

Chirality can be present in polymer molecules in two ways: either the molecule adopts a chiral conformation, for instance a onehanded helix, or either many molecules, which typically adopt a planar, achiral conformation, stack in a chiral (one-handed 10 helical) way. Examples of conjugated polymers that can adopt a helical conformation are cis-transoid poly(acetylene)s, ${ }^{5,6}$ poly(3,6-carbazole)s, ${ }^{7}$ gallic acid substituted poly(dithienopyrrole)s, ${ }^{8}$ oxazoline-functionalized poly(thiophene)s, ${ }^{9}$ poly(3,6-polyphenanthrene)s, ${ }^{10}$ etc. If chiral side-chains are 15 implanted, one helical sense can be discriminated and will prevail. Also conjugated foldamers, such as $\operatorname{oligo}(m-$ phenyleneethynylene) quite often adopt a helical conformation in particular conditions. ${ }^{11-13}$ However, most conjugated polymers show a clear tendency to aggregate. Typically, the polymers 20 adopt a more planar conformation and $\pi$-interactions promote stacking of the polymer chains. The planarization and $\pi$-stacking is commonly accompanied by a redshift. If the polymers are substituted with chiral side-chains, the asymmetric branching results in a nonparallel orientation of the stacked polymer chains.

${ }_{25}$ The use of enantiomerically pure chiral substituents can again result in a preferred helical sense. A typical example of such polymer is chirally substituted regioregular HT-coupled P3AT. ${ }^{14-}$ 18 In good solvent, the polymer adopts a random-coil conformation, in which chirality is not expressed. In poor 30 solvents or film, however, the polymer chains planarize and stack, resulting in a strong redshift $(\sim 80 \mathrm{~nm})$. Moreover, strong Cotton effects become visible, reflecting the (supramolecular) chirality. Also many other chiral conjugated polymers display this behavior.

\section{${ }_{35}$ Chiral expression}

Optical expressions of chirality, such as optical rotation and CD, originate from an electric and magnetic transition dipole moment both being nonzero and pointing in the same direction (or a nonzero component pointing in the same direction). ${ }^{19}$ As a 40 consequence, a helical electron displacement, that is inevitably associated with a helical conjugated backbone, fulfils these requirements and gives rise to chiral optical effects. Indeed, a typical (monosignate) Cotton effect, centred at the absorption maximum of the particular transition, is observed in the CD 45 spectrum of these systems. As the relative orientation of the electric and magnetic transition dipole moments changes sign when the opposite handedness is considered (i.e. when the other enantiomer is considered), the sign of the Cotton effect is reversed. In contrast, two transition dipole moments, originating 50 from a linear electron displacement, can couple. This is for instance found when conjugated polymers stack: the transition dipole moments are located along the polymer backbone and can electrostatically interact (exciton coupling). Alternatively, this can also be the case when two segments of a helical backbone 55 become chirally oriented. The original transition is split. When the two polymer chains are rotated towards each other, which is the case when chiral substituents are employed, also the transition dipole moments are chirally oriented. The electron displacement, associated with the transition dipole moment, of one chain results 60 in a circular displacement in the other and vice versa. The chiral alignment of the transition dipole moments thus provides a magnetic dipole moment to the transitions. As a consequence, the two transitions that originate from the exciton coupling are both provided by a monosignate Cotton effect. Since the chiral 65 orientation for both transitions is opposite, the two monosignate Cotton effects have a different sign as well. The overall Cotton effect, which is the sum of both effects, is a bisignate Cotton effect. The occurrence of such Cotton effects is summarized in Figure 1.

70 In order to "quantify" and compare the chiral response of (a) given system(s), one can define the g-value, which equals

$$
g_{a b s}=\frac{2\left(A_{L}-A_{R}\right)}{A_{L}+A_{R}}
$$

where $A_{L}$ and $A_{R}$ denote for the absorption of left and right circularly polarized light, respectively. If the Cotton effects are 75 measured in solution, a concentration independency of the Cotton effects points at (macro)molecular chirality. As aggregationinduced supramolecular chirality depends on the formation of the chiral superstructure, it is therefore concentration dependent. Furthermore, the chirality can be "intensive", which means that it 80 only depends on the chiral organization on the site where light is absorbed. ${ }^{20}$ This results in $\mathrm{g}_{\mathrm{abs}}$-values that are independent on the thickness of the film studied, as found in poly(thiophene)s. ${ }^{21,18}$ Conversely, in "extensive" chirality, as typically observed in chiral poly(fluorene)s forming (long range) chlolesteric liquid85 crystalline states, the origin of the chiroptical effect is extrinsic to the site of photon absorption and the $\mathrm{g}_{\mathrm{abs}}$-value varies with film thickness. $^{22}$

The sign of a bisignate cotton effect depends on the orientated of the stacked polymer chains (lef and right handed). Since 90 conjugated polymers can aggregate differently in different solvents, this can result in a inversion of the sign of the Cotton effect in different solvents. ${ }^{23}$

\section{Conjugated copolymers}

\section{Random copolymers}

95 In this part chiral random copolymers composed of chiral and achiral monomers are considered. Helical polymers containing chiral and achiral units randomly distributed along the polymer chain are known to show sergeants-and-soldiers behavior. This principle was first discovered by Green in poly(isocyanates). ${ }^{24}$ 100 Cooperativity results in a fraction of one-handed helices that is much higher than the fraction of chiral monomers, resulting in a positive nonlinear relationship between chiral properties (optical rotation, circular dichroism ...) and the fraction of chiral 

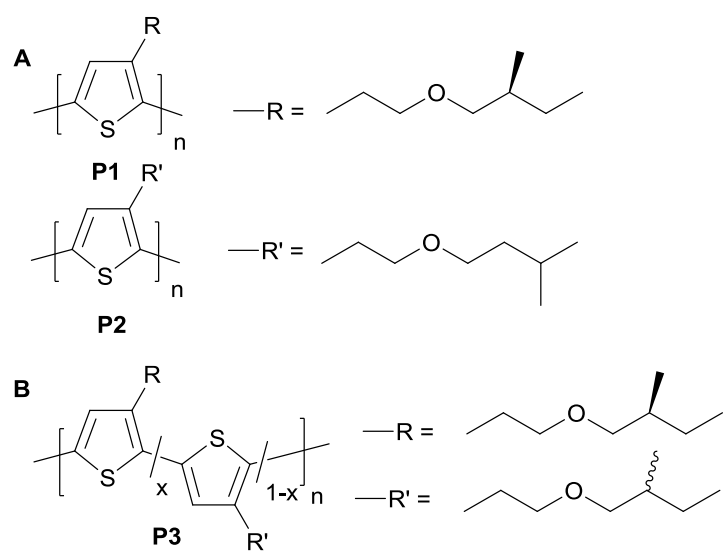

Figure 2. Structure of the poly(3-alkylthiophene)s.

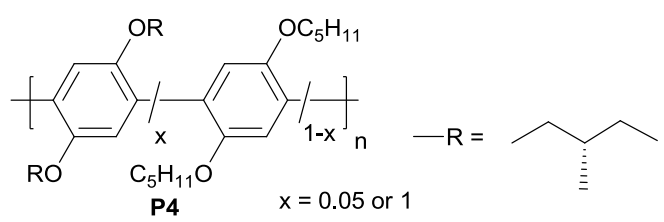

Figure 3. Structure of the poly(phenylene)s.
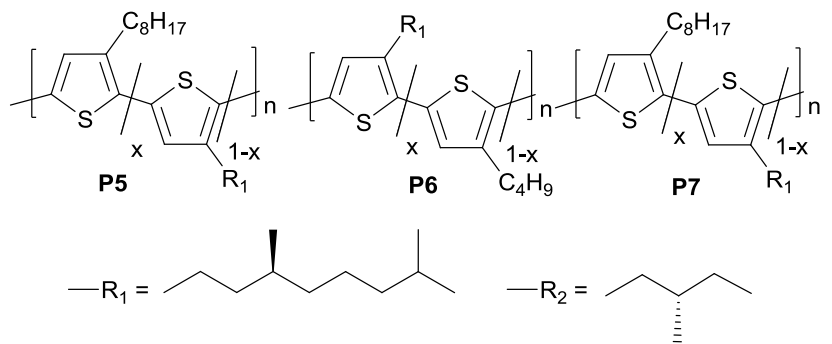

Figure 4. Structure of the poly(3-alkylthiophene)s.

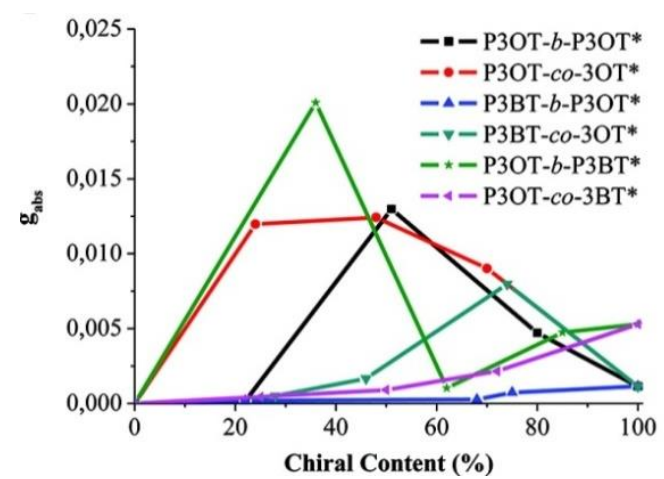

Figure 5. Sergeants-and-soldier behavior in copoly(3-alkylthiophene)s. Reprinted with permission from ref. 26. Copyright 2011 American Chemical Society.

monomers. Related to the sergeants-and-soldiers principle Green also discovered the 'majority rules' effect. ${ }^{25}$ This can be found in copolymers of $R$ and $S$ enantiomers of a chiral monomer. The excess of one enantiomer results in a large energy difference 15 between both helical senses and, consequently, the chiral properties depend nonlinearly on the enantiomeric excess of the monomer.

Both principles require a chiral superstructure in which the building blocks, i.c. the monomers, are randomly built in. The 20 most straightforward way is a helical polymer composed of chiral and achiral monomers (sergeants-and-soldiers) or both enantiomers of a chiral monomer (majority rules).

Also poly(3-alkylthiophene)s (P3ATs) have been used in a sergeants-and-soldiers experiment (Figure 2). ${ }^{27}$ Aggregates of

${ }_{25}$ P3ATs are composed of planar, stacked polymer chains; in case of chiral substituents, the polymer chains stack in a chiral (onehanded helical) way. Therefore, sergeants-and-soldiers behavior can be found if helical stacks are made composed of chiral and achiral homopolymers randomly incorporated into the helical 30 stacks. The experiments confirmed sergeants-and-soldiers behavior, albeit not as clear as in for instance poly(isocyanates). The origin of the weaker effect might be the difference in nature of the substituent and concomitant aggregation behavior of the different homopolymers (P1 and P2). Indeed, perfect sergeants35 and-soldiers behavior requires that both components are equally well built in. The difference in side-chain results in a different solubility, while the respective homopolymers are only randomly incorporated if the two homopolymers have exactly the same solubility. This was confirmed by mixing the two polymers and

40 let them aggregate. Depending on the molar mass, either the achiral or chiral polymer aggregates first, acting as seeds for the second homopolymer. Consequently, if the chiral homopolymer aggregates first, it enforces its chirality to the second homopolymer, resulting in a chiral response that is higher than 45 expected on the fraction of chiral homopolymer. Conversely, if the achiral homopolymer aggregates first, the chiral expression is less. In a second experiment (Figure 2B), the authors copolymerized $R$ and $S$ enantiomers of a chiral monomer (P3). The chiral aggregation of the resulting copolymers was evaluated 50 in 1-decanol at room temperature. A strong nonlinear relation between the g-value $(=\Delta \varepsilon / \varepsilon)$ and the enantiomeric excess was observed, pointing at a kind of majority rules behavior.

Fiesel et al. have prepared a copoly(phenylene) composed of 5\% chiral monomers and $95 \%$ achiral monomers and compared the 55 g-value with that of the corresponding chiral homopolymer (Figure 3, P4) ${ }^{28}$ From the fact that the copolymer already showed a significant chiral response, it was concluded that sergeant-andsoldiers behavior is present.

In an at first sight analogous experiment Verswyvel et al. have 60 synthesized series of copoly(3-alkylthiophenes) composed of chiral and achiral monomers, in which the nature of the chiral and achiral side-chains was varied (Figure 4, P5-P7). ${ }^{26}$ Surprisingly, for most polymers the g-value did not nonlinear and continuously increase, as expected in sergeants-and-soldiers behavior, but 65 instead showed an optimum at $<100 \%$ chiral monomer (Figure 5). This behavior was explained by assuming that, compared with the chiral homopolymer, the molecular asymmetry of copolymers results in a smaller tendency of the polymer chains to stack in parallel fashion (i.e. the $\pi$-interactions are weakened), resulting in 70 a larger angle by which the polymer chains are rotated (i.e. the effect of the asymmetrical branching of side-chain becomes stronger). Note that these experiments are not in contradiction with the report of Fiesel et al, since the latter series is only composed of the chiral homopolymer and a copolymer containing 75 only $5 \%$ chiral monomers.

If all reported measurements are taken together, it is clear that care should be taken with the interpretation of the chiroptical measurements and the attribution of the sergeants-and-soldiers 

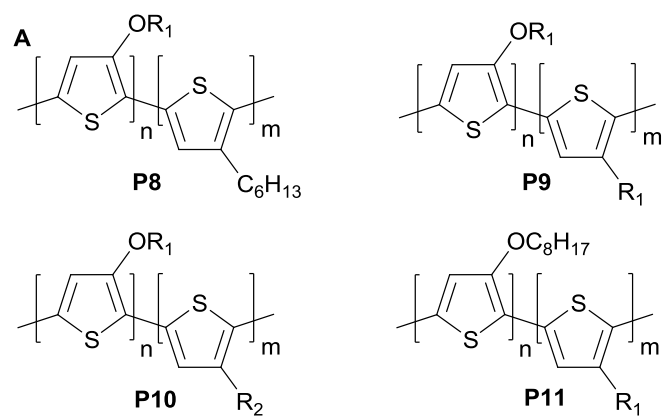

B

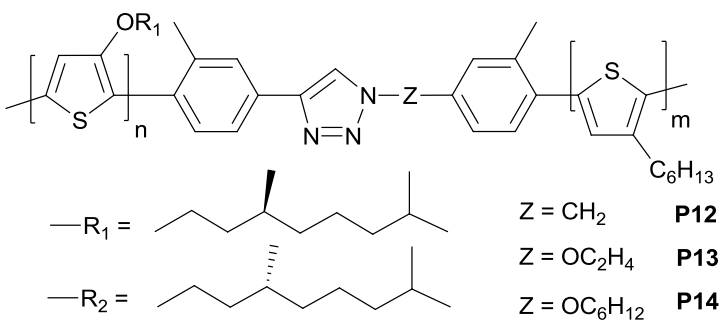

Figure 6. Structure of the P3AT- $b$-P3AOT block copolymers: A) without spacer and B) with spacer.

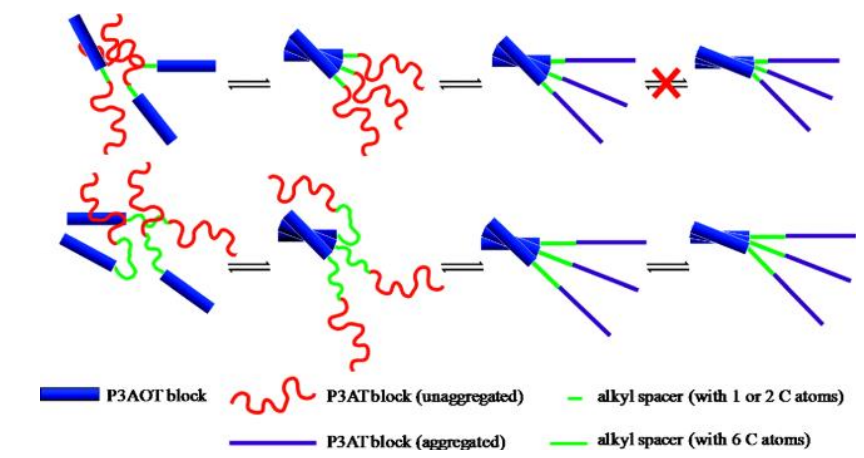

Figure 7. Schematic representation of the aggregation behaviour of P3AOT- $b$-P3AT block copolymers. Reprinted with permission from ref. 29. Copyright 2011 American Chemical Society.

and majority-rules principles in aggregated conjugated polymers. ${ }_{10}$ Truly sergeants-and-soldiers and majority-rules experiments require the formation of a chiral (helical) superstructure composed of chiral and achiral components or $R$ and $S$ components. This corresponds to the stacking of chiral and achiral homopolymers and $R$ and $S$ homopolymers, respectively.

15 Moreover, both polymers should have the same solubility. The aggregation of copolymers is more complicated, since this in fact concerns different chiral entities, i.e. different "sergeants".

\section{Block copolymers}

Van den Bergh et al. have studied several block copolymers 20 composed of a HT-P3AT and a HT-coupled poly(3alkoxythiophene) (P3AOT) block (Figure 6). A first series consisted of block-copolymers in which both blocks were directly connected to each other. ${ }^{30,31}$ These polymers were prepared by successive monomer addition using the Ni(dppp)-mediated chain25 growth polymerization. Four different block copolymers were prepared: P8-P11 (Figure 6A). The chiral properties of the polymers were evaluated by gradually adding nonsolvent to a solution of the polymer and monitoring the changes in the UV-vis and CD spectra. Since P3AT and P3AOT absorb at significantly 30 different wavelengths (P3AT: $\lambda_{\max } \sim 450 \mathrm{~nm}$ (good solvent), $\sim 510$ $\mathrm{nm}$ (poor solvent); P3AOT: $\lambda_{\max } \sim 600 \mathrm{~nm}$ (good solvent), $\sim 610$ $\mathrm{nm}$ (poor solvent)), the UV-vis and CD spectra provide clear information on the blocks which are (chirally) stacked. The length of the P3AT and P3AOT block were kept more or less 35 constant throughout the series. Since P3AOT has a poorer solubility, this block aggregates first upon decreasing the solvent quality.

It was found that the block aggregating first imposes restrictions on the second block; in most cases, the first block enforces its

40 aggregation to the second block. Indeed, if the P3AOT-block is substituted with a chiral $(S)$-substituent, the chiral, right-handed stacking of the P3AOTs is preserved in the stacking of the P3ATs, irrespective of the nature of the alkyl substituent. Indeed, the P3AT block in P8, stacks in a chiral way. Moreover, in P9, 45 that contains a $(S)$-P3AT block of which the homopolymer stacks in a left-handed helical fashion, the P3AT block stacks in a righthanded helical way. The most surprising results were found in P11. Upon decreasing the solvent quality, first the P3AOT block stacks. Being substituted with achiral side-chains, the chains 50 stack parallel. When the solvent quality is further decreased, the P3ATs are forced to stack. The aggregated P3AOT block does not allow a chiral stacking, while the asymmetric branching prevents a parallel stacking. Instead, the P3AT chains adopt a helical conformation, as is evidenced by the presence of a 55 monosignate Cotton effect in the CD spectra.

From these experiments, it becomes clear that the first block can pose limitations on the aggregation behavior of the second block, resulting in macro- and supramolecular properties which are not found in the corresponding homopolymers.

60 A next series of P3AOT- $b$-P3AT block copolymers studied were composed of a $(S)$-P3AOT and a P3HT block, separated by a flexible alkyl spacer of different length $\left(\mathrm{CH}_{2}(\mathbf{P 1 2}), \mathrm{OC}_{2} \mathrm{H}_{4}(\mathbf{P 1 3})\right.$ and $\mathrm{OC}_{6} \mathrm{H}_{12}$ (P14)) (Figure 6B). ${ }^{29}$ The polymers were prepared in a two-step process. First, the homopolymers were prepared 65 starting from a functionalized Ni-initiator. In a second stage, the P3AOT block equipped with and acetylene group was clicked to the P3HT functionalized with an azide.

In a series of experiments related to the previous ones, it was investigated whether the P3AOT block can impose its chiral 70 stacking to the (achiral) P3HT block when connected via a flexible alkyl spacer. Also in these experiments, the solvent quality was gradually decreased. The experiments revealed that the P3AT block, although being achirally substituted, stacks in a chiral way, showing that the P3AOT block imposed its 75 supramolecular structure to the P3AT block. Moreover, the magnitude of the $\mathrm{CD}$ spectra showed no difference in intensity for the block copolymers with different spacer lengths. Interestingly, the experiments showed that the P3AT block in the block copolymer with the longest alkyl spacer showed 80 reorganization at high nonsolvent quality: it adopts a supramolecular structure in which the stacked polymer chains become rotated with a smaller angle and display a smaller chiral response. This reorganization is not observed in block copolymers with shorter or no alkyl group. This summarized in ${ }_{85}$ Figure 7.

Also block copolymers composed of a chiral and achiral P3AT 


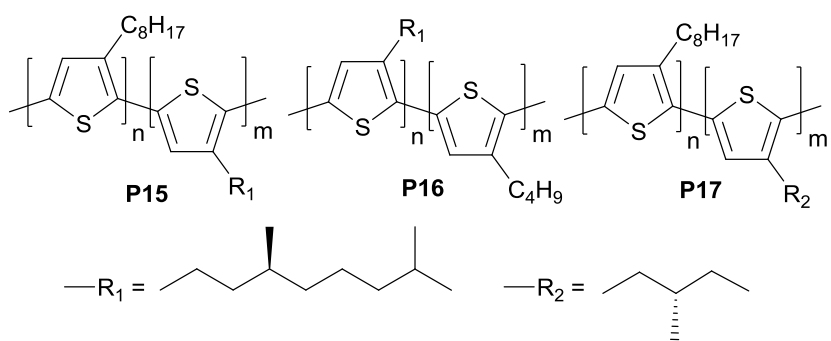

Figure 8. Structure of the chiral P3AT block copolymers.

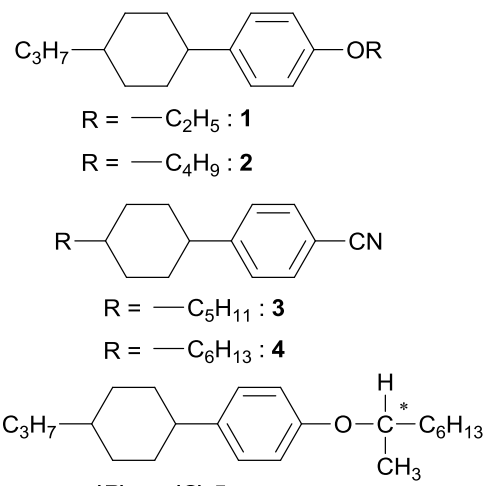

$(R)$ - or $(S)-5$
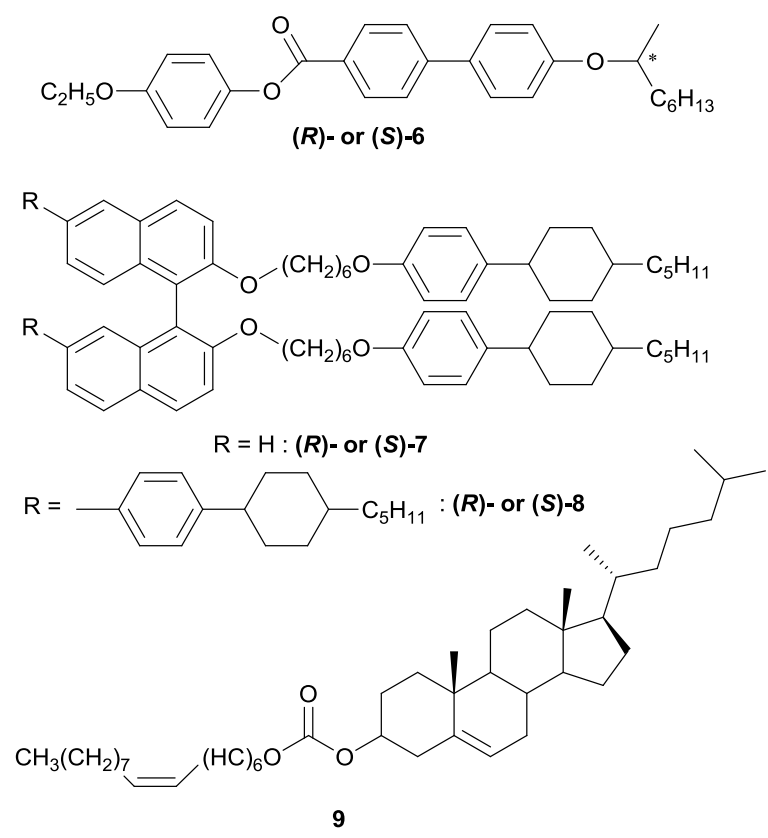

Figure 9. Structures of the liquid crystals and chiral dopants for N*-LC.

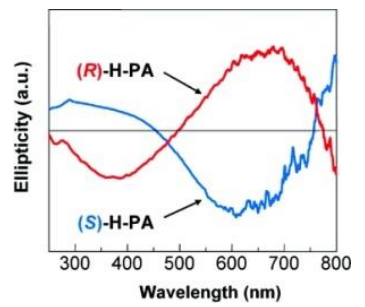

Figure 10. CD spectra of PA thin films $((R)-\mathrm{H}-\mathrm{PA}$ and $(S)-\mathrm{H}-\mathrm{PA})$ synthesized in $(R)-\mathrm{N}^{*}$-LC and $(S)$-N*-LC, respectively. Reprinted with permission from ref. 32. Copyright 2010 American Chemical Society.
10 block have been prepared and studied (P15-P17, Figure 8) ${ }^{26}$ The polymers were also prepared by successive monomer addition. The block copolymer differs in the length of the respective blocks and the nature (length) of the alkyl substituent, keeping the overall degree of polymerization constant. Aggregation occurs at 15 lower nonsolvent content as substituents are shorter and less branched and/or the block is longer. It was found that if the blocks aggregate separately, the block aggregating first imposes its supramolecular structure to the second one (Figure 5). If, however, the blocks do not show a significant difference in 20 solubility, they form mixed aggregates. Similar to random copolymers, the highest chiral response is not found for the polymer which the highest fraction of chiral side-chains (i.e. the homopolymer), but polymers with an intermediate amount of chiral monomers.

\section{${ }_{25}$ Noncovalent chiral interaction}

Besides the ability to induce optical activity by means of a covalent bond between the polymer and a chiral fragment as described above, also a noncovalent interaction between both can result in a chiral expression. Here we can differentiate between 30 the synthesis of chiral polymers (i) in a chiral solvent or (ii) by interaction of a chiral moiety with the main chain or (iii) with the functionalized side chains, (iv) complexation of conjugated oligomers equipped with ligands around metal cores resulting in conjugated polymers and (v) coordination of conjugated 35 polymers around metals by interaction of their side-chain or main chain with these metals. To restrict the scope of this review, metal-containing conjugated polymers are not discussed and reference is made to other reviews. $5,9,34-37$

\section{Chirality induced by chiral solvents}

40 Although polymerization of achiral monomers in an isotropic chiral solvent does not result in a chiral polymer, however it is possible in a liquid-crystal (LC) solvent in its chiral nematic (cholesteric) phase $\left(\mathrm{N}^{*}\right.$-LC). The $\mathrm{N}^{*}$-LC field provides a chiral pattern as a molecular mold and the polymer grows in this chiral 45 field to form a one-handed helical structure. The $\mathrm{N}^{*}$-LC is obtained by mixing nematic liquid crystals, typically phenylcyclohexyl derivatives $\mathbf{1}, \mathbf{2}, 3$ or 4 with a chiral dopant 7 (). The latter compound is in general a derivative of binaphthalene which transfers its chiral twist through the solvent, 50 hereby applying a kind of a sergeants-and-soldiers principle. Apart from this, chiral liquid crystals 5 or $\mathbf{6}$ were also reported which made the chiral dopant redundant. The helical pitch of the helical nematic solvent, and therefore also of the resulting polymer chains, can depend on the nature, the purity and the 55 concentration of the chiral dopant. ${ }^{32,38}$ A chiral dopant with aromatic groups directly on the 6 and 6' positions of binaphthalene rings, $\mathbf{8}$, resulting in an extremely strong twisting power is reported. ${ }^{39}$ Temperature and stirring speed are essential parameters for holding the medium in its $\mathrm{N}^{*}$-LC state during the 60 polymerization. Already in 1986, the group of Shirakawa described the first synthesis of poly(acetylene) (PA) in a $\mathrm{N}^{*}$-LC solvent instead of a normal isotropic solvent. ${ }^{40}$ To induce polymerization, acetylene gas was guided through the reaction vessel with a $\mathrm{N}^{*}$-LC reaction medium and a Ziegler-Natta 65 catalyst. ${ }^{32,41-45}$ This was quickly followed by other chiral 

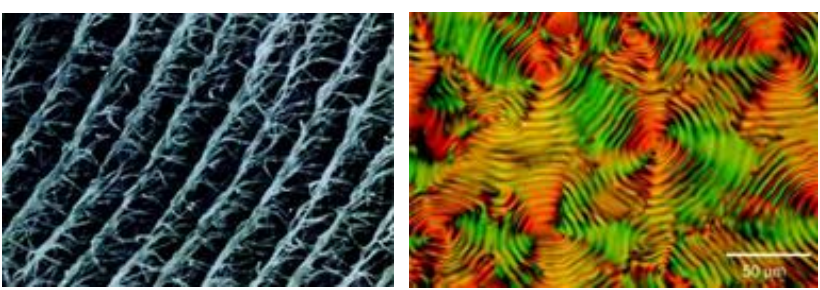

Figure 11. Left: SEM micrographs $(4 \mu \mathrm{m})$ of poly(acetylene) film synthesized in (R)-N*-LC. Right: POM of $\mathrm{N}^{*}$-LC. Reprinted with permission from ref. 33. Copyright 2009 American Chemical Society.

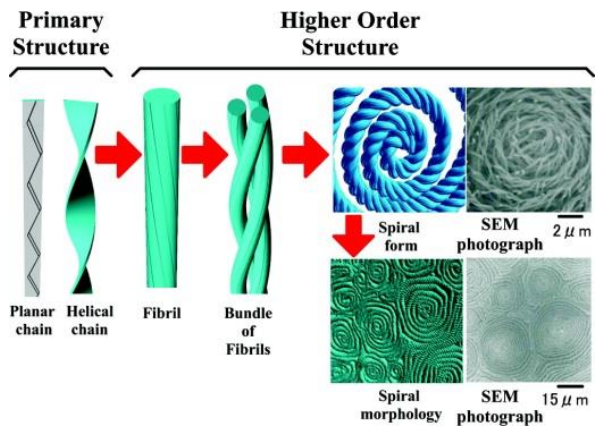

Figure 12. Hierarchical helical structures of PA. Reprinted with permission from ref. 33. Copyright 2009 American Chemical Society.

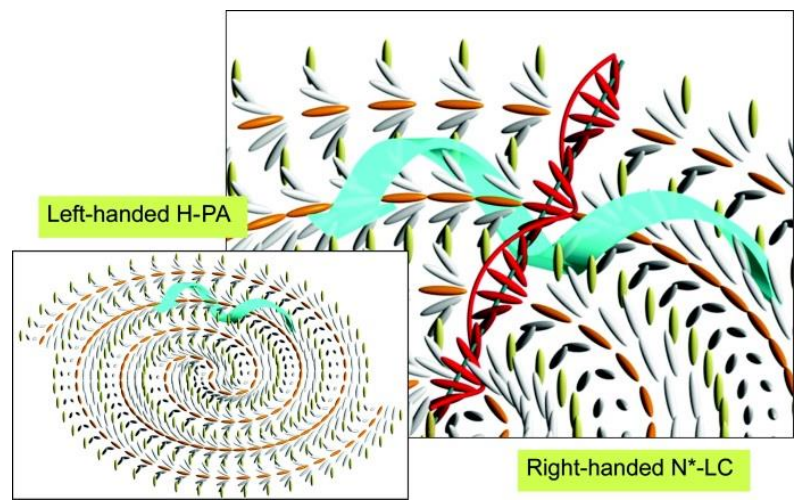

Figure 13. Schematic representation of the orientation of the LCs and the 10 PA during the polymerization. Reprinted with permission from ref. 33. Copyright 2009 American Chemical Society.

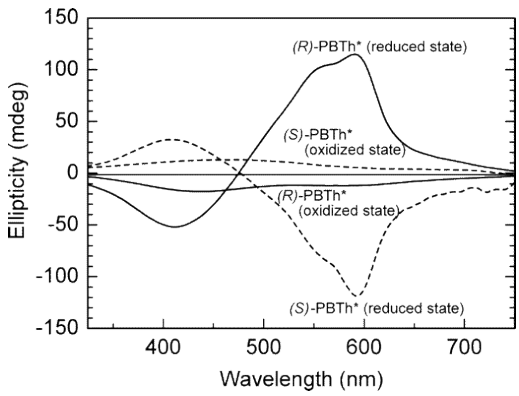

Figure 14. $(R)$ - and $(S)$-PBT films in oxidized and reduced states. The oxidized and reduced states of the polymer were produced by application 15 of potential (+1.3 V: oxidized state; $0.1 \mathrm{~V}$ : reduced state vs. $\left.\mathrm{Ag} / \mathrm{Ag}^{+}\right)$in $0.1 \mathrm{M}$ TBAP/acetonitrile solution. Reprinted with permission from ref. 49. Copyright 2005 American Chemical Society.

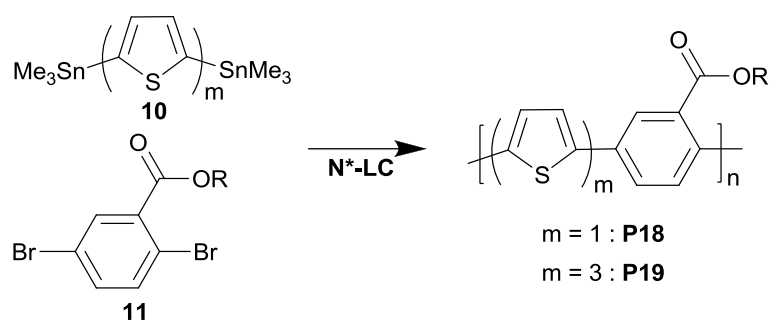

Figure 15. Synthesis of PT derivatives via Stille coupling.

20 conjugated polymers like poly(3,4-ethylenedioxythiophene) (PEDOT) ${ }^{46-48}$ poly(bithiophene) (PBT) ${ }^{49}$ poly(pyrrole) (PPy), ${ }^{50}$ poly(para-phenylenevinylene) (PPE $)^{51}$ and copolymers of those $^{52-56}$ starting from achiral monomers. CD measurements of the resulting films and solutions all show a bisignate Cotton 25 effect in the region of the $\pi-\pi^{*}$ transition ( 450 to $800 \mathrm{~nm}$ ) of the conjugated backbone, and there is a mirror image relationship when the other enantiomer of the solvent is used (). It was observed that the typical fingerprint picture of the $\mathrm{N}^{*}$-LC solvent under polarized optical microscopy (POM) is similarly 30 adopted by the resulting polymer (). Clearly, the helical PA mimics the helical structure of the chiral nematic LCs in the solvent and the helical chains are further bundled through Van der Waals interactions to form helical fibrils compiled together in bundles ().

${ }_{35}$ Due to the insolubility and infusibility of the unsubstituted polymers, the helical structure is retained even after heating or washing with toluene. ${ }^{41}$ Control experiments of the polymerization in the absence of a chiral dopant or liquid crystals in the solvent or polymerization above the $\mathrm{N}^{*}$-LC temperature all 40 show a randomly oriented fibrillar morphology, rather than the helical one for the resulting polymer.

Akagi et al. investigated the structural relationship between the $\mathrm{N}^{*}$-LC solvent and the resulting chiral PA. ${ }^{32,43,44}$ They elucidated that the distance between the fibril bundles of the helical PA is 45 nearly equal to half the helical pitch of the $\mathrm{N}^{*}$-LC. Even more, the helical axes of the solvent and the PA are oriented orthogonal to each other and, more remarkable, the helical twist direction of the resulted PA is opposite to that of the chiral solvent. All observations were explained by a comprehensive model for the 50 spiral morphology presented in . A more extended reviews about helical PA synthesized in $\mathrm{N}^{*}$-LC solvents is written by Akagi. ${ }^{33,57}$ When oxidizing the chiral films, the bisignate effect changed to a monosignate effect and the intensity weakens or the sign of the CD signal can even invert (). This was ascribed to an intercalation 55 of the perchlorate ions between the main chains by the redox process which partially breaks the chiral aggregation. ${ }^{46}$ The polymer films significantly change color during oxidation. The reversible inversion of the sign of the $\mathrm{CD}$ in the redox process, accompanied by the color change, demonstrates that the chirality 60 of the polymer can be changed by applying a voltage. The material can therefore be used as an electrochromic material, i.e. it is possible to control chirality of the polymer film by applying an external potential using an electrochemical technique.

Alternating chiral copolymers of achiral unsubstituted thiophene 65 and phenylene resulting from a Stille polycondensation or electrochemical polymerization in a $\mathrm{N}^{*}$-LC were presented by 


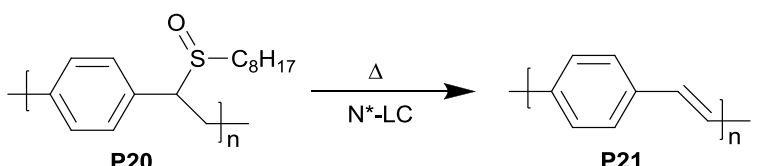

Figure 16. Synthetic route for PPV.

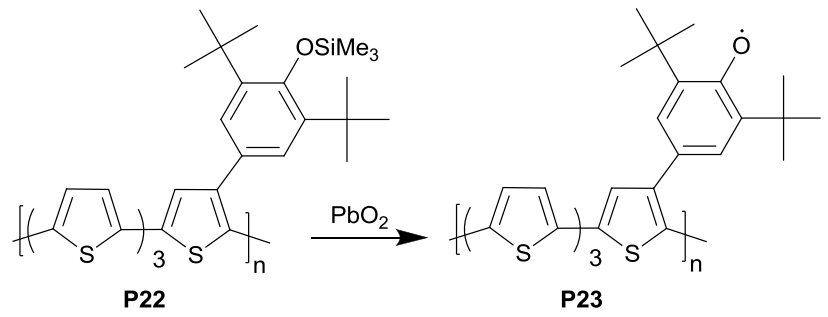

Figure 17. Conversion from the chiral precursor polyradical to the actual 5

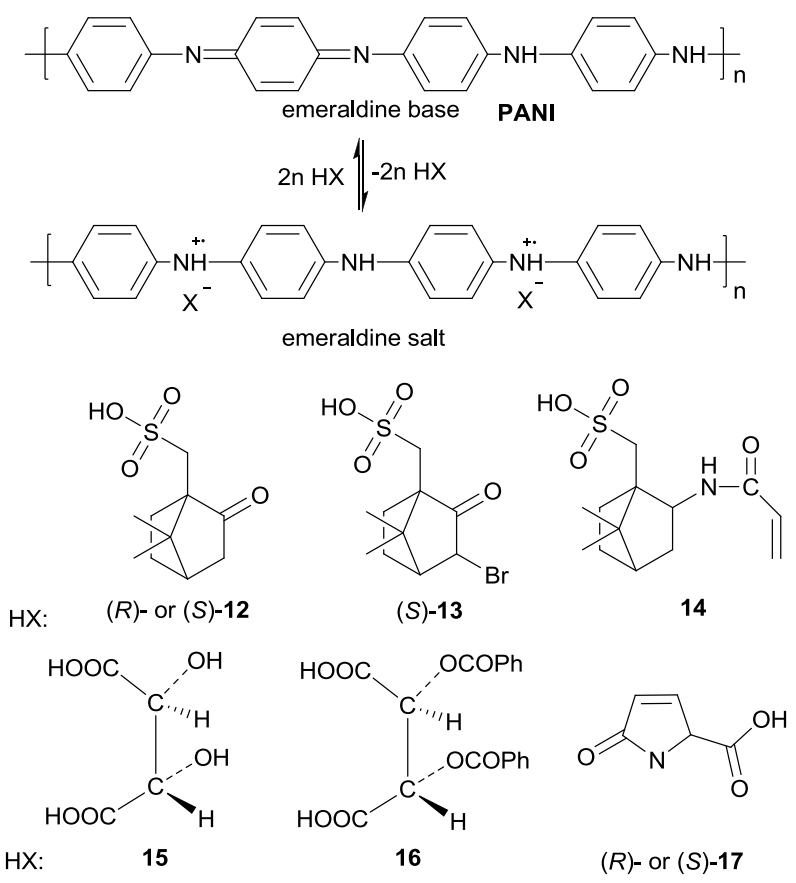

Figure 18. Structure of the parent emeraldine base and the doped emeraldine salt and several common chiral dopants 12-17.

${ }_{10}$ Goto and Akagi. ${ }^{52-56}$ The polymers preserve their chiral structure in an isotropic solution, even after dissolution and washing. This was attributed to the steric hindrance of the ester groups at the 2position of the benzene rings which stabilizes the chiral structure of the polymer. However, heat treatment of the polymer in a 15 cumene solution for $24 \mathrm{~h}$ at $150{ }^{\circ} \mathrm{C}$ resulted in a significant decrease in $\mathrm{CD}$ intensity. Besides this, alternating copolymers with one and three thiophene units between the phenyl units were polymerized from 10 and 11 (, P18 and P19). ${ }^{55}$ While the polymer with three thiophene units, P19, preserves its helical 20 order after removal of the $\mathrm{N}^{*} \mathrm{LC}$ and dissolution in $\mathrm{CHCl}_{3}$ or THF, the one with only one thiophene per repeating unit, P18, does not. This was ascribed to the higher solubility of these polymers, resulting in a loss of chiral aggregation upon dissolution in $\mathrm{CHCl}_{3}$ or THF.

${ }_{25}$ The preparation of chiral unsubstituted PPV films follows a slightly different approach as the CPs described above. A sulfonium precursor polymer $\mathbf{P 2 0}$ is made prior to the actual PPV (P21) formation (). The former is dissolved in a chiral cholesteric liquid crystal matrix, $(R)$ - or $(S)-6$, which increases the 30 temperature of the $\mathrm{N}^{*}$-LC phase of the solvent needed for the next step in the synthesis. The inherent chirality of the liquid crystal avoids the need for a chiral dopant. Heating of the reaction mixture to $105-110{ }^{\circ} \mathrm{C}$ eliminates the sulfonium and results in a chiral PPV* film with only a weak Cotton effect in the $\pi-\pi^{*}$ 35 transition of the PPV main chain $(402 \mathrm{~nm})$ in the CD spectra. Due to the high viscosity of the precursor polymer, the low-viscous cholesteric liquid mold was not able to fully penetrate the precursor PPV. Consequently, the ordered chiral structure was not imparted on the entire PPV film and only the top surface

40 adopts the chiral order which explains the weak signal. This again refers to the importance of a careful consideration of the affinity of the precursor polymer for the $\mathrm{N}^{*}$-LC as well as its solubility therein for the success of the conversion to the actual polymer. This chirality-transfer approach was also used by Goto to reduce 45 the required reaction steps often seen in the synthesis of chiral conjugated polymers bearing stable radical groups. ${ }^{60}$ The chiral trimethylsilane radical precursor P22, synthesized in $(R)$ - or $(S)-6$ as a $\mathrm{N}^{*}$-LC phase, delivers the chiral polyradical $\mathbf{P 2 3}$ after deprotection with acid and oxidation with $\mathrm{PbO}_{2}$ (). It was shown 50 that the chirality of the polymers was maintained upon oxidation with $\mathrm{PbO}_{2}$.

During the progress in this field, new methods to obtain a chiral reaction field have been appeared. Liquid crystallinity can also be induced by some natural polymers in water. Akagi and Goto 55 reported a lyotropic $\mathrm{N}^{*}$-LC solution of $20 \mathrm{wt}$. \% of double stranded DNA of salmon sperm in water. ${ }^{48}$ Note that this polymerization is exploited in an aquatic medium instead of an organic solvent. The conjugated polymer grows between the twisted main chains of DNA, forming a one-handed helical 60 structure. Also a concentrated water solution of hydroxypropyl cellulose as a polymer lyotropic liquid crystal was presented and provided a $\mathrm{N}^{*}$-LC medium for right-handed helices. ${ }^{46} \mathrm{~A}$ new, conveniently available cholesteric derivate, 9, was used as a chiral inducer for the synthesis of chiral pyridine-based 65 conjugated polymers. ${ }^{61}$

\section{Chirality by chiral interaction with the main chain}

Interaction between achiral monomers/polymers and a chiral moiety in an isotropic solution can also result in optically active polymers. The chiral particles can be present during the 70 polymerization or can convert a pre-existing achiral polymer to a chiral one. To allow electrostatic interaction, the polymer backbones are electrochemically charged or doped with an acid. Unsubstituted poly(aniline) (PANI) and derivatives are the most studied materials in this field. ${ }^{58,62-73,59,74-88}$ Their emeraldine base 75 (dark green) is typically doped with the chiral $(S)$ - or $(R)$ camphor sulfonic acid $((S)$ - or $(R)$-12) to the corresponding optically active emeraldine salt (blue)

(). ${ }^{58,63,65,66,68,69,72,73,59,74-87}$ Therefore the PANI base is dissolved in NMP, DMF, DMSO or $\mathrm{CHCl}_{3}$ and enantiomerically pure $(R)$ - or $80(S)$-12 was added. CD spectroscopy of the doped PANI in solution or film shows various $\mathrm{CD}$ bands associated with the absorption bands in UV-vis, indicating a chiral conformation of the resulting chain. ${ }^{68,76}$ A mirror image 

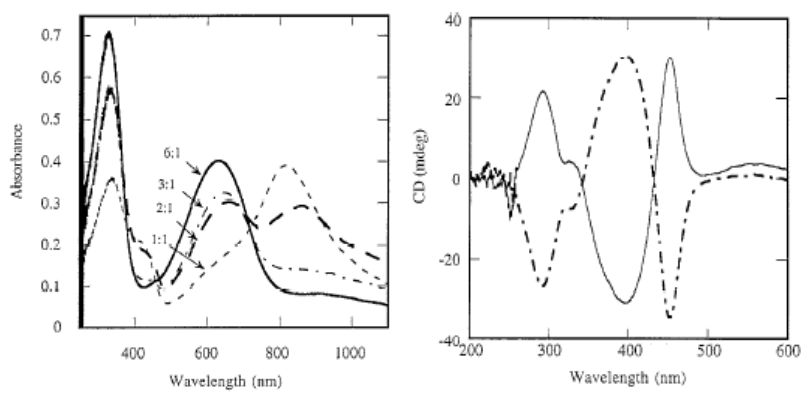

Figure 19. Left: UV-vis spectrum op $(R)$-doped PANI in NMP with a PANI/12 molar ratio ranger from 6:1 to 1:1. Right: CD spectrum of $(R)$ and $(S)$ - doped PANI in NMP (dashed line is $(S)$-doped; solid line is $(R)$ 5 doped). Reprinted with permission from ref. 58. Copyright 1999 Elsevier.

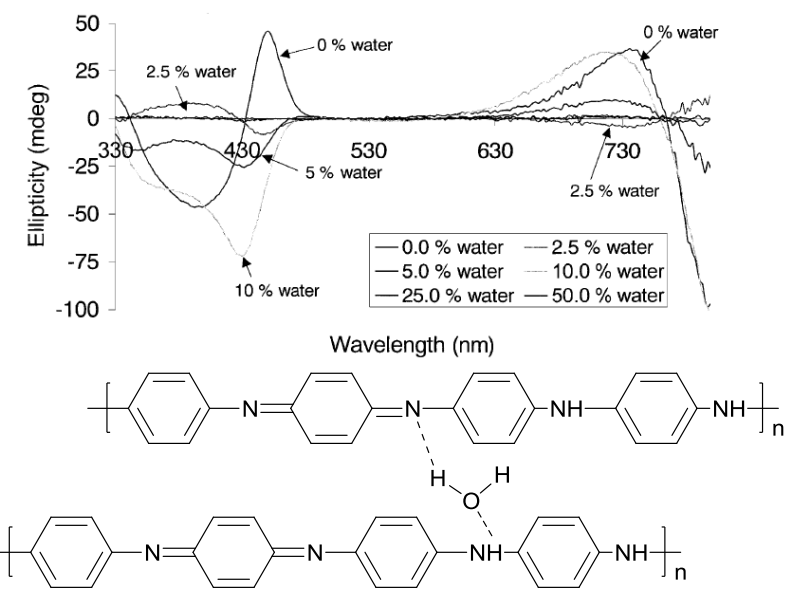

Figure 20. Effect of initially added water on the CD signal of 12-induced PANI in NMP. Reprinted with permission from ref. 59. Copyright 2003 Elsevier.
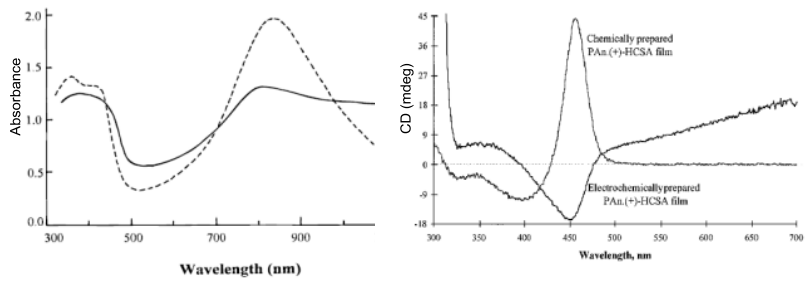

Figure 21. UV-vis (left) and CD- (right) spectra of chemically (dashed line) and electrochemically prepared PANI (solid line). Reprinted with permission from ref. 85. Copyright 1998 American Chemical Society.

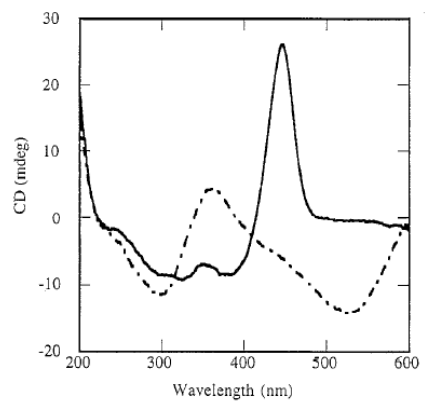

15 Figure 22. CD-spectra of 12-doped (solid line) and dedoped (dashed line) PANI films. Reprinted with permission from ref. 58. Copyright 1999 Elsevier.

relationship is observed between $(R)-\mathbf{1 2}$ in comparison with the $(S)-\mathbf{1 2}$ as a chiral dopant (). During the doping process, the chiral
20 dopants arrange themselves around the conjugated chains by electrostatic interactions and $\mathrm{H}$-bonds (between a hydrogen of a $\mathrm{NH}+-$ group and the ketone functionality of 12). ${ }^{69}$ In this way they can give rise to a structure that is very similar to that of a conjugated polymer with covalent attached chiral pendants. ${ }^{77}$ The 25 groups of Wallace and Kaner noticed remarkable changes in the resulting CD signals of chiral doped PANI, in some cases even an inversion of configuration, when small amounts of water (1 to $10 \% \mathrm{v} / \mathrm{v})$ were added in the organic solvents prior to the acid doping of PANI with 12 (). ${ }^{59,74}$ This effects may arise from prior $30 \mathrm{H}$-bonding of the emeraldine base by water which compete with the polymer-amine H-bonds. To bind $\mathbf{1 2}$ anyhow, a different conformation is needed, leading to the opposite hand of the PANI helix ().

Apart from mixing emeraldine base with a chiral dopant, chiral, 35 doped PANI can also be obtained by (chemically or electrochemically) polymerizing aniline in the presence of a chiral dopant. ${ }^{83,84}$ Norris et al. investigated the conformational differences between the chemical and electrochemically prepared PANI and the influence on the UV-vis en CD spectra. ${ }^{85}$ It was 40 elucidated that the chemically prepared chiral PANI appear in a "compact coil" conformation with an intense, well-defined localized polaron band at ca. $825 \mathrm{~nm}$ in UV-vis (). In contrast, this polaron band was weaker and broader in the electrochemically produced film and a free-carrier tail appeared 45 in the near infrared which corresponds to a significant electron delocalization. The latter suggests a more "expanded coil" type conformation of the polymer chain. Also in CD-spectroscopy both conformations differ significantly (). Heating PANI in the "expanded coil" state above $140{ }^{\circ} \mathrm{C}$ shifts the UV-vis and CD 50 spectra to the characteristics of the "compact coil" state. This state is retained, even after cooling of the sample, demonstrating that the "compact coil" state is the more thermodynamic stable form. Heating below $130{ }^{\circ} \mathrm{C}$ and subsequent cooling of the "expanded coil" PANI showed a normal thermochromic behavior 55 with recovery of the original UV-vis and CD spectra. Besides the electropolymerization of PANI, also enzymes can catalyse the synthesis of optically active PANI without the need of any chiral dopant. $^{65,66}$

Besides $\mathbf{1 2}$ as a chiral dopant, also other chiral acids can be used. ${ }_{60}$ Wallace et al. presented a chemical preparation of doped PANI from the emeraldine base with (1S)-(+)-3-bromocamphor-10sulfonic acid $(S-13)$ or a acrylamide sulfonic acid derivative (14) (). ${ }^{69,70}$ The interaction with one of both acids and PANI generates a similar UV-vis and CD spectra to that reported for PANI/12 65 salts. The presence of the same binding sites $\left(\mathrm{SO}_{3}{ }^{-}\right.$and a carbonyl site) explains the similarity in their conformation and the resulting chiroptical data. Interestingly, doping of PANI with tartaric acid (15) demonstrates that these latter structural features are not essential for chiral induction in the PANI chain. Even 16 70 was able to undergo enantioselective interaction with PANI, indicating that the carboxylic ester group in tartaric acid is not a critical component. Finally, Yang et al. used the enantiomers $(S)$ (-)- and (R)-(+)-2-pyrrolidone-5-carboylic acid $((S)-17)$ and $(R)$ 17) to synthesize chiral nanotubes of PANI. ${ }^{70,71,80}$

${ }_{75}$ Since the doping/dedoping of PANI is simple acid/base chemistry, dopants are easily removed with a base. When the 


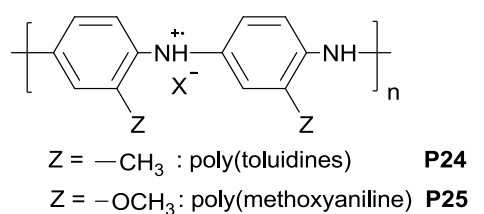

Figure 23. Chemical structure of common substituted PANI derivatives.

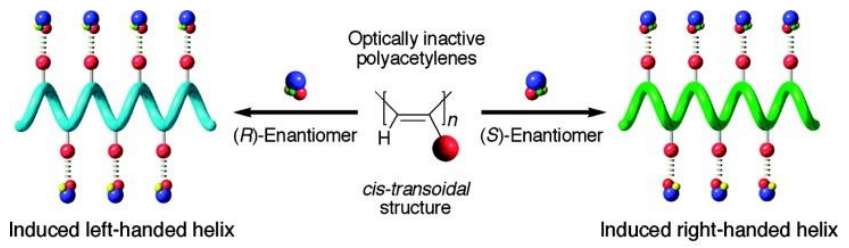

Figure 24. Structural model of the helicity induction in 5 functionalized poly(acetylene)s. Reprinted with permission from ref. 89. Copyright 2008 American Chemical Society.<smiles>C=CC(C)C</smiles>

P26

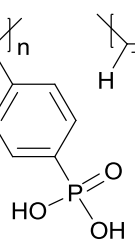

P27<smiles>CC=CCCCCCC(C)C</smiles>

P28<smiles>C=CC(=C)C(=O)NC(=C)C</smiles>

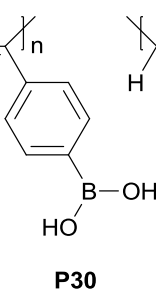<smiles>CC=C(C)c1cccc(NCC)c1</smiles>

P31

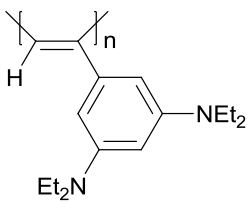

P32

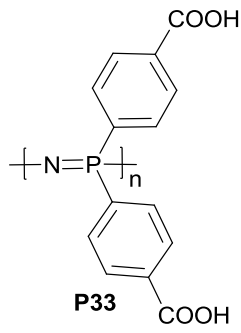

Figure 25. Structures of poly(phenylacetylene) derivatives and poly(phosphazene).

10 chiral, doped PANI-films are dedoped with $\mathrm{NH}_{4} \mathrm{OH}$, the chirality remains despite the chiral counter ions are removed. ${ }^{58}$ Even redoping of the resulting emeraldine base with $\mathrm{HCl}$ retains the optical activity. This was shown by the occurrence of typical CD bands of the PANI polymers, although the signals appear at 15 different wavelengths compared to the doped form (). The retention of the chiral structure was attributed to the intramolecular electrostatic interactions and hydrogen bonds in the PANI polymer. Wallace revealed that changing to very polar solvents that can break H-bonds, such as $m$-cresol, destroys the 20 helical structure. ${ }^{68,69,77}$ Apart from chiroptical effects, the chirality in PANI is also manifested as chiral hosts. The threedimensional cavities are able to selectively recognize and bind chiral molecules with the right size and shape. Therefore it can discriminate between the enantiomers of a chiral amino acid. ${ }_{25}{ }^{58,67,72,78,79,81,87}$ Apart from unsubstituted PANI, also the substituted PANI chains have been evaluated for their ability for chiral induction. ${ }^{62-64,68,69,76}$ The enantioselective doping process of poly(toluidine) (P24) or poly (o-methoxyaniline) (P25) (methyl or methoxy

30 pendants) () in combination with $(S)-\mathbf{1 2}$ in DMSO or DMF is substantially slower than with the parent PANI but results in similar CD spectra as the latter. The presence of the methyl or methoxy groups ortho or meta to the nitrogen sterically

hinders, and therefore slows down, the incorporation of chiral 1235 ions in a helical conformation. ${ }^{68,69}$ Also the electropolymerization of these polymers results in a "compact coil" conformation. This is in contrast to the electropolymerization of the parent PANI/12 which delivers chains in an "expanded coil" situation (vide supra). The chiral conformation is likewise retained after 40 dedoping with base and (eventually) redoping with $\mathrm{HCl}$. It is interesting that the chiral conformations were maintained in both aprotic and protic solvents.

Similar chiral doping of the polymer backbone can be applied on poly(pyrrole)s $\quad$ (PPy). ${ }^{74-78}$ Pyrrole was electrochemically 45 polymerized in aqueous solution containing D- or L-tartaric acid, D- or L- glutamic acid, L-aspartic acid, $(S)$ - or $(R)$ - 12 or L-lactic acid. ${ }^{92,94,95}$ The resulting chiral PPy films exhibited CD spectra with a mirror image relationship depending on the enantiomer used, each possessing two main $\mathrm{CD}$ bands in the visible region at 50680 and $720 \mathrm{~nm}$. The strength of the acid-base interaction between the different chiral acids mentioned above slightly changes the CD spectra in shape.

PPy nanotubes doped with $(R)$ - or $(S)-\mathbf{1 2}$ are dedoped by the application of a constant voltage of $-0.6 \mathrm{~V}$ for $10 \mathrm{~min} .^{94} \mathrm{In}$ this 55 dedoped state, the nanotubes are able to discriminate between $\mathrm{D}$ and L- phenylalanine, similarly to what was observed for the dedoped PANI/12. Overoxidation, realized by applying a large positive potential of chiral PPy films prepared in the presence of D- or L- glutamic acid or D- and L- aspartic acid, results in the 60 formation of carbonyl and carboxyl groups in the polymer structure and elimination of the dopants. The resulting film acts as a porous electrode coating, which has cation-exchange and molecular sieving properties when a voltage was applied. The films selectively retain the ${ }^{91} \mathrm{D}$ - or L- glutamic acid or D- or L65 aspartic acid that was used during the doping process in their history. This stereoselective recognition during a redox cycle was also reported before by the group of Roncali for a chiral poly(thiophene) interacting with $13 .{ }^{14}$

\section{Chirality by chiral interaction with the side chains}

70 Noncovalent interactions between chiral moieties and functional groups in the polymer's side-chains, instead of with the main chain, can also be used in order to introduce chirality in conjugated polymers (). Charges in the side chains mainly originate from acidic or basic groups. Various 75 poly(phenylacetylene)s with versatile functional side chains were extensively investigated by the group of Yashima (Figure 25). He reported the formation of one-handed helices from achiral polymers by acid-base complexation of chiral amines, amino alcohols and amino acids with organic acid functions in the side 80 chains. $^{96,90,97-107,89,108,109}$ During this interaction, the achiral cistransoid $\mathbf{P 2 6}$ in DMSO or in film acts as dynamic helix with helix reversal every 2-4 monomer units . During interaction with various optically active amines and amino alcohols, the polymeramine complexes results in single-handed helices which reflect 
the stereochemistry of the used amines. ${ }^{96,90,97,102,103}$ During this

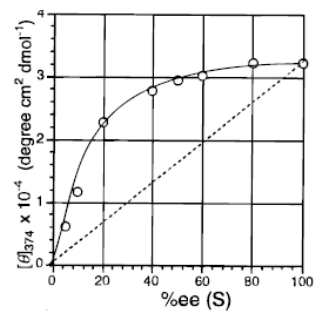

Figure 26. Left: $\mathrm{CD}$ intensities in function of the enantiomeric excess of $(S)$-2-amino-propane-1-ol in a non-racemic mixture in DMSO showing a 5 sergeants-and-soldiers effect. Reprinted with permission from ref. 90. Copyright 1997 American Chemical Society.

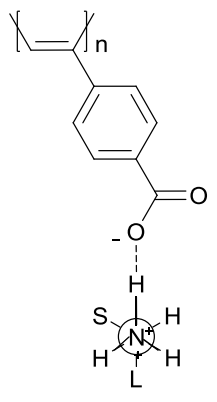

(R)-Amine

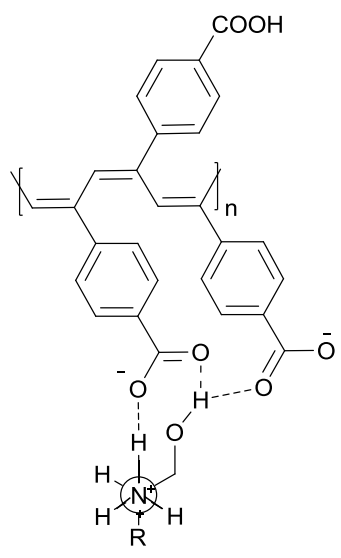

(R)-Amino alcohol
Figure 27. Interaction of $\mathbf{P 2 6}$ with an $(R)$-amine and an $(R)$-amino alcohol. Reprinted with permission from ref. 90. Copyright 1997 American Chemical Society.

process, the rather irregular twist of the adjacent double bonds around a single bond is transformed into the helical conformation with a predominant handedness. This is confirmed by a redshift of the polymer backbone absorption and a bisignate Cotton15 effect, which shows a mirror image relationship between both enantiomers. A unique positive nonlinear relationship is observed in a non-racemic mixture between the enantiomeric excess of amines or amino alcohols and the CD signals (). The effect is greater for the amino alcohols than for the amines (vide infra). ${ }_{20}$ Furthermore, addition of a small amount of chiral amines to a P26-achiral amine complex causes a dramatic increase in the magnitude of the Cotton effect. These chirality amplifications are similar to the majority rules and sergeants-and-soldiers principle, respectively.

${ }_{25}$ Even more, the Cotton-effects appear to increase with an increased bulkiness of the chiral amine and a decrease is noticed going from primary amine > secondary amine >> tertiary amine. In addition, secondary and tertiary amines show opposite CD signals compared to the primary amines when their 30 configurations are the same. Going from amines to amino alcohols dramatically increases the CD signals, irrespective of the bulkiness of the amino alcohols. This implies that, besides the acid-base interaction, also hydrogen bond formation of the hydroxyl group to a carboxy moiety of the polymers must play an 35 important role (). During the complexation, the bulkiest substituent (L) is placed remote from the polymer (antistaggered) and the smaller alkyl group (S) and the hydrogen are in a staggered position. Therefore the complexes with $(R)$ - and $(S)$ - amines show mirror image CD signals.

${ }_{40}$ Because the complexation of the amines and the amino alcohols to the polymer are acid-base interactions, also the basicity of the chiral inducers plays an important role and reflects the binding affinity. Since the complex formation and ion dissociation are dependent on the polarity of the solvent, the intensity of the CD

45 effects also depends on the solvent. In a $\mathrm{CHCl}_{3} / \mathrm{DMSO}$ mixture, an increased $\mathrm{CD}$ signal is noticed compared with that in neat DMSO, DMF or $\mathrm{CH}_{3} \mathrm{CN} / \mathrm{DMSO}$ mixture. In the more polar DMSO, DMF and $\mathrm{CH}_{3} \mathrm{CN}$, ion dissociation is stronger than in less polar $\mathrm{CHCl}_{3}$ resulting in a strong interaction in the latter 50 solvent. This also implies that the optical activity disappears when the polymer-amine complexes are exposed to stronger acids (e.g. trifluoroacetic acids) than the carboxy acids in the side chain. When the complexed chiral amines are replaced by various achiral amines, the helical configuration can even be memorized, 55 i.e. the one-handed screw sense of the helices is retained. ${ }^{97,103}$

The aforementioned behavior of the P26 is also evaluated in aqueous solutions to allow interaction with natural amino acids and other biomolecules. ${ }^{99,105,106}$ In order to provide solubility of P26 in water, the salt of the polymer was made. Interestingly, 60 only a very small amount of chiral amino acid is needed to induce chirality in these polymer salts. The protonation of the amine functions of the biomolecules, and therefore the interaction with the polymer, can be controlled by the $\mathrm{pH}$. Anionic polyelectrolytes interact with the positively charged ammonium 65 groups of the amino acids through electrostatic attractive interactions, whereas the electrostatic repulsion between the anionic polymers and the carboxylate groups of the amino acids disturb the effective complexation, resulting in a $\mathrm{pH}$ optimum. Inspired by the phosphate interaction of nucleic acids with a 70 variety of biomolecules, also achiral cis-transoid P27 and its monoethyl ester P28 was synthesized. ${ }^{100,104,105,107,108}$ Chirality induction by interactions with chiral biomolecules such as the backbone of a polypeptide main chain as well as the terminal and side chain amino groups, 1,2- or 1,3-amino alcohol residues and 75 1,2-diols were reported in DMSO and water. Moreover, interaction between the salt of $\mathbf{P 2 8}$ and the more peculiar $\mathrm{N}$ methylfulleropyrrolidine (a $\mathrm{C}_{60}$-derivative) in DMSO-water mixtures also results in a predominant one-handed helix. ${ }^{110}$ The phosphonic ester $\mathbf{P 2 8}$ is the most sensitive toward chiral 80 induction because these polymers have the most bulky side groups which generate a steric hindrance between the neighbouring pendants. This creates a steeper internal rotation energy barrier for the polymer backbone and may make the polymer chain more rigid with a longer persistent length, and ${ }_{85}$ correlated smaller amount of reversals, than those of the other polymers. Complexation of $\mathbf{P 2 8}$ and the pharmaceutically important chiral 3-substituted pyrrolidines and piperazines in DMSO and water allow a formation of nematic and cholesteric LC phases in concentrated water solutions, correlated to the 90 significantly high main chain stiffness, and this results in an increased amplification of the macromolecular helicity. ${ }^{108}$ The phosphonic acid groups on $\mathbf{P 2 7}$ and $\mathbf{P 2 8}$ are more acidic than the carboxy groups of $\mathbf{P 2 6}$ and therefore they respond more efficiently to chiral amines which results in a more intense CD 95 signal. Changing now to the stronger sulfonic acid groups (P29) 
initially decreases the signal in CD spectroscopy because of

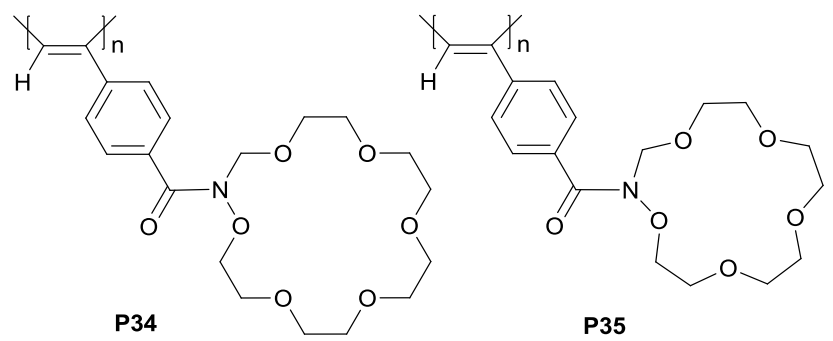

Figure 28. Poly(acetylene) with aza-crown-ether pendants.

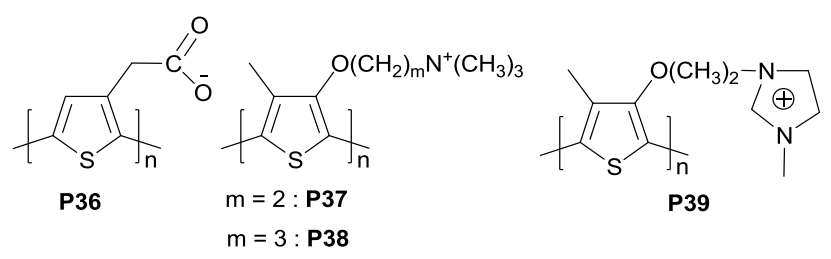

Figure 29. Molecular structures of functionalized PTs.

dissociation of the ions preventing a proper chiral induction. ${ }^{105-}$ 107 This dissociation can be suppressed by addition of a common salt of the chiral substance (for instance with $p$-toluene sulfonic 10 acid). In that way, the ion pair complex is preserved, which is reflected by an extreme increase in CD signal, much higher than for P28. A very stable memory effect of this sulfonic acid bearing derivatives is observed when chiral amines are replaced by achiral diamines.

15 The interaction between carboxyphenyl groups and chiral amines was also observed for other polymer backbones than poly(acetylene). Maeda et al. reported the chirality induction between the achiral and flexible $\mathbf{P 3 3}$ and chiral amines. ${ }^{98,109}$ Even more, the chirality was retained after the addition of the opposite 20 enantiomer of the amine. Also a chiral filter effect towards amino acids was described. The polymers exhibit a chiral recognition ability, leading to the enantioselective adsorption of one of the enantiomers. This was not observed for the P26.

Vandeleene et al. reported poly(phenylene ethynylene-alt25 bithiophene)s copolymers with chiral pendants and pendants bearing carboxylic acid groups in solution and in film. ${ }^{111}$ Here, it was noticed that the addition of chiral primary amines results in chiral aggregation of the polymers. When the chiral centres of the pendants and the amines were the same, cooperation between 30 both in the helical stacking was observed in CD spectroscopy. The opposite holds when they are opposed. Even more, a nonlinear relationship, i.e. majority rules principle, was observed between both.

Taking the above acid-base interactions into account, an obvious 35 next step is to exchange both functions: the amine in the side chains and the organic acid as a chiral inducer. Various acyclic and cyclic, primary, secondary and tertiary amines were introduced in the side chains of the poly(acetylene)s. ${ }^{112-116}$ The same acid-base chemistry as above holds for the interaction of 40 these polymers with chiral carboxylic acids. However, the resulting optical activity is strongly related to the structure of the amine functionalized polymer. For instance, when the optical activity of P31 and P32 induced by chiral acid are compared, completely different spectra are observed. ${ }^{115}$ The UV-vis 45 spectrum of P32 shows an increased redshift compared to P31, suggesting that a helical structure with a long conjugation length is formed. More surprisingly, the P32 shows no induced CD spectrum in the presence of optically active carboxylic acids in THF while this is present in combination with P31. In the less 50 polar $\mathrm{CHCl}_{3}$, a stronger interaction between the amino group and the acid is present and chirality is also induced in P32. The stronger interacting $\alpha$-hydroxy acids can however be used in THF to generate a predominantly one-handed helix with P32. This $\alpha$ hydroxy group may participate in the intermolecular hydrogen 55 bonding with the amino group on the $\mathbf{P 3 2}$, together with the acidbase ion pairing of the carboxy group. It is also this hydrogen bond that is responsible for the opposite sign in CD spectroscopy if acids or $\alpha$-hydroxy acids with the same chirality are complexed with P31.

${ }_{60}$ Likewise, complexation of achiral P30 with various kinds of acyclic and cyclic chiral molecules with two or more functional groups including carbohydrates, steroids, nucleosides and (oligo)nucleotides induces a prevailing helical conformation confirmed by a bisignate Cotton effect in CD spectroscopy. ${ }^{117,118}$ 65 The interacting groups can be an interacting amine and alcohol function, two alcohol functions or two amine functions.

The side chains can also be equipped with crown ethers. ${ }^{119-122}$ When aza-18-crown-6 ethers are present in the pendants (, P34), a predominantly one-handed helical conformation upon 70 complexation with various chiral compounds, such as amino acids, peptides, aminosugars, amines, and amino alcohols was formed in aqueous solution $\left(\mathrm{HClO}_{4 \mathrm{aq}}\right) .{ }^{119}$ Except for L-cysteine and L-phenylalanine, all the CD spectra of the 19 common free L-amino acids gave the same pattern. Adding $\mathrm{NaCl}$ or $\mathrm{KCl}$ also ${ }_{75}$ decreases the magnitude of the CD spectra, which suggests the importance of the crown ether-ammonium complexation in acidic water. The interactions between the amino acids and the crown ethers are stronger in aprotic solvents than in protic ones because the latter disturb the hydrogen bonds between the ammonium 80 group and the oxygen and/or the nitrogen atoms in the crown ether. ${ }^{121}$ The high sensitivity of the crown ethers toward amino acids result in detection limits less than $5 \mathrm{mg} / \mathrm{mL}$ or $0.005 \%$ of enantiomeric excess in $\mathrm{CH}_{3} \mathrm{CN} .{ }^{120,121}$ This type of side chains also responds to chiral amino alcohols and follows the majority 85 rules principle. Changing to the aza-15-crown-5 ether (P35) increases the sensitivity for the secondary amino acids but weakens the nonlinear chiral amplifications in case of enantiomeric excesses.

Not only poly(acetylene) derivatives were appended with 90 functionalized side chains for chiral interactions. Moreover, poly(thiophene) (PT) was functionalized with negatively charged carboxylic acid groups or positively charged amine groups attached as side chains to allow interaction with chiral peptides, ${ }^{123}$ amino acids, ${ }^{124}$ polysaccharides ${ }^{125}$ and nucleic ${ }_{95}$ acids $^{126-132}$. When the water-soluble poly(thiophene acetic acid) (P36) () is dissolved in an alkaline buffer, the pendants become deprotonated and planarization of the polymer backbone occurs. ${ }^{123}$ This is attributed to the loss of the hydrogen bonding between the adjacent carboxyl groups and the electrostatic 100 repulsion between the negative charges. After the addition of $1-$ 2 eq. of a positively charged peptide (due to the lysine groups in 
the side chains), the absorption maximum in UV-vis spectroscopy is blue-shifted which refers to a decrease of the effective

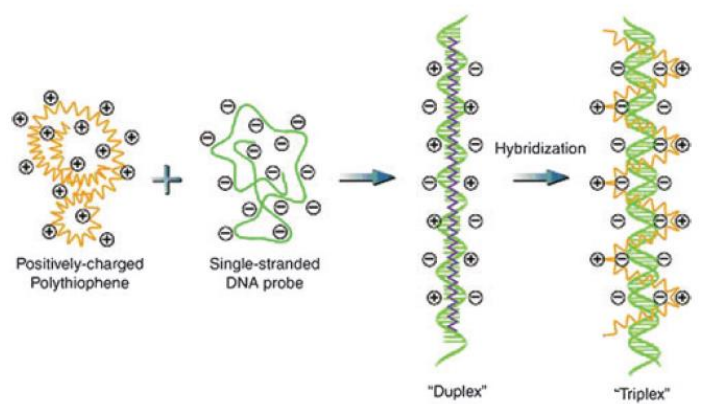

Figure 30. Schematic description of the poly(thiophene)/single-stranded 5 nucleic acid duplex and poly(thiophene)/hybridized nucleic acid triplex. Reprinted with permission from ref. 131.

conjugation length of the polymer backbone. This demonstrates that the interaction between the negative P36 and the positively charged peptide disrupts the electrostatic repulsion and forces the 10 polymer backbone to adopt a more nonplanar, helical hetero duplex. Concomitantly, the peptide shifts from a random-coil conformation to this new helical form. The supramolecular organization is confirmed by the appearance of a bisignate Cotton effect in CD spectroscopy. Fluorescence spectroscopy supports 15 this conformational change by a clear blue shift and an increase in intensity of the emission spectrum after the addition of the peptide. The former indicates again the transition of the PT to the random-coil conformation and the latter that the separation of the polymer chains occurs which inhibits the nonradiative relaxation.

${ }_{20}$ Changing the negative carboxylic acid groups to positively charged ammonium groups affords the interaction with negatively charged chiral inducers. An often used polymer with this characteristics is the poly(3-alkoxy-4-methylthiophene) (P37 \& P38) (). ${ }^{124,126,127}$ Mixing the conjugated achiral P37

25 or P38 with a folic acid forces the polymers in optically active supramolecular aggregates, resulting in a distinct color change from yellow to purple, a pronounced fluorescence quenching and the appearance of a bisignate induced CD spectrum. ${ }^{124}$ The extent of the color change depends strongly on the polymer used 30 whereby $\mathbf{P 3 7}$ seems to provoke the strongest interactions. P38, on its turn, is more preferred in combination with adenosine triphosphate (ATP) as chiral inducer instead of folic acid. Shortening the spacer between the ammonium and the PT backbone can increase the hydrophilicity. Considering the facts 35 that P37 and ATP are more hydrophilic in comparison with P38 and folic acid, respectively, the specificity of the P37 towards folic acid sensing is straightforward. Indeed, the interactions are related to the hydrophilic/hydrophobic balance between the PT cation and the bio anion. Since $\mathbf{P 3 8}$ is more hydrophobic and easy 40 to aggregate with both folic acid and ATP, the selectivity disappears. On the other hand, the higher efficiency of the ATPP37 interaction is not strong enough to result in highly ordered aggregates. Both reasons favor the selectivity of P37 toward folic acid. Even more, the solvent has an enormous effect on the CD 45 intensities. Using an optimized solvent mixture of water/methanol $5 / 5$ weakens the $\pi-\pi$ stacking of the PT backbones because of the solubility improvement of both the quaternary ammonium groups in water and of the hydrophobic moieties in methanol. Also the added amount of ATP, in comparison the polymer, can alter the 50 intensity and even the sign of the CD spectrum. ${ }^{127}$

The water-soluble $\mathbf{P 3 8}$ was as well used in combination with the natural polysaccharide schizophyllan (SPG). ${ }^{125}$ This saccharide is known to exist as a right-handed triple helix in water but as a single random coil in DMSO. Adding water to a solution of P38 55 and SPG in DMSO will force the saccharide in the helical conformation around the PT backbone, which acts as a charged molecular wire. A redshift in UV-vis spectroscopy, without any vibronic bands, and an intense bisignate Cotton effect in the $\pi-\pi^{*}$ transition region of the PT was observed, indicating a helical 60 structure of the PT. Emission spectra show a $41 \mathrm{~nm}$ redshift as well, accompanied by a slight increase in intensity. This observation suggests a more planar, morre isolated and more insulated molecular wire of PT may be formed during the complexation with SPG, resulting in reduction of the interchain 65 interactions.

Nucleic acids tend to induce chirality in achiral P38 and P39 (). ${ }^{128-132}$ The yellow solution of the random coil PTs becomes red after the addition of one equivalent of negatively charged oligonucleotide, because of the formation of a so-called duplex 70 between both (). This complex tends to be insoluble in the medium in which they are formed and this is confirmed by similar absorption spectra to that of the PT in solid state. When mixing the complementary oligonucleotide to this mixture, the solutions become yellow again. Indeed, a more soluble charged 75 complex, a triplex, is formed. This complex contains a righthanded helically oriented PT, as is shown in CD measurements. When the complementary oligonucleotide has one or two mismatches, a very distinct, stable and reproducible UV-vis spectrum is observed. These observations are again supported by ${ }_{80}$ fluorescence measurements. The same approach, with similar results, was found for a cationic poly(fluorene-co-phenylene) copolymerized with 2,1,3-benzothiadiazole chromophore to induce two emission colors. ${ }^{133}$

This chiral induction of aforementioned biomolecules, and the 85 resulting spectroscopic alternations, can also be used for easy, simple and non-destructive sensing applications of these moieties with detection limits up to five molecules in $3 \mathrm{~mL} .{ }^{129}$

\section{Conclusions}

Chirality in systems that are based on conjugated polymers and 90 composed of multiple components - two different block (block copolymers), two different monomers (random copolymers), a polymer prepared in a chiral medium or a polymer in the presence of a chiral additive - show a chiroptical behavior that fundamentally differs from systems in which the chiral 95 component is absent. Moreover, the presence of multiple components can give rise to a unique behavior, that cannot be realized in systems based on single components. The progress in the synthesis of other multicomponent systems might access even more advanced, chiral structures and inferred responses, such as 100 conjugated polymers attached to chiral/patterned surfaces or nanoparticles, etc.

\section{Notes and references}


${ }^{a}$ Laboratory of Polymer Synthesis, Celestijnenlaan 200F, 3001 Heverlee, Belgium. Fax: +32(0)16/32.79.90; Tel: +32(0)16/32.74.20; E-mail: guy.koeckelberghs@chem.kuleuven.be

1. L. A. P. Kane-Maguire and G. G. Wallace, Chem Soc Rev, 2010, 39, 2545-2576.

2. Y. Cheng, L. Chen, J. Song, X. Zou, and T. Liu, Polym. J., 2005, 37, 355-362.

3. L. Pu, Chem. Rev., 1998, 98, 2405-2494.

4. J.-P. Lère-Porte, J. J. E. Moreau, F. Serein-Spirau, and S. Wakim, Chem. Commun., 2002, 3020-3021.

5. E. Yashima, K. Maeda, H. Iida, Y. Furusho, and K. Nagai, Chem. Rev., 2009, 109, 6102-211.

6. E. Yashima, K. Maeda, and Y. Furusho, Acc. Chem. Res., 2008, 41, 1166-80.

15 7. Z.-B. Zhang, M. Motonaga, M. Fujiki, and C. E. McKenna, Macromolecules, 2003, 36, 6956-6958.

8. W. Vanormelingen, K. Van den Bergh, T. Verbiest, and G. Koeckelberghs, Macromolecules, 2008, 41, 5582-5589.

9. H. Goto, Y. Okamoto, and E. Yashima, Chem. Eur. J., 2002, 8, 40274036.

10. W. Vanormelingen, A. Smeets, E. Franz, I. Asselberghs, K. Clays, T. Verbiest, and G. Koeckelberghs, Macromolecules, 2009, 42, 42824287.

11. R. B. Prince, J. G. Saven, P. G. Wolynes, and J. S. Moore, J. Am. Chem. Soc., 1999, 121, 3114-3121.

12. P.-J. Prest, R. B. Prince, and J. S. Moore, J. Am. Chem. Soc., 1999, 121, 5933-5939.

13. J. C. Nelson, J. G. Saven, J. S. Moore, and P. G. Wolynes, Science, 1997, 277, 1793-1796.

30 14. M. Lemaire, D. Delabouglise, R. Garreau, A. Guy, and J. Roncali, J. Chem. Soc., Chem. Commun., 1988, 658.

15. B. M. W. Langeveld-Voss, D. Beljonne, Z. Shuai, R. A. J. Janssen, S. C. J. Meskers, E. W. Meijer, and J.-L. Brédas, Adv. Mater., 1998, 10, $1343-1348$

35 16. M. M. Bouman, E. E. Havinga, R. A. J. Janssen, and E. W. Meijer, Mol. Cryst. Liq. Cryst., 1994, 256, 439-448.

17. G. Bidan, S. Guillerez, and V. Sorokin, Adv. Mater., 1996, 8, $157-$ 160 .

18. G. Lakhwani, G. Koeckelberghs, S. C. J. Meskers, and R. A. J. 40 Janssen, Chem. Phys. Lett. , 2007, 437, 193-197.

19. N. Berova, K. Nakanishi, and R. W. Woody, Eds., Circular Dichroism: Principles and Applications, Wiley, 2nd edn., 2000.

20. G. Lakhwani, J. Gielen, M. Kemerink, P. C. M. Christianen, R. A. J. Janssen, and S. C. J. Meskers, J Phys Chem B, 2009, 113, 14047-51.

45 21. S. Vandeleene, K. Van den Bergh, T. Verbiest, and G. Koeckelberghs, Macromolecules, 2008, 41, 5123-5131.

22. M. R. Craig, P. Jonkheijm, S. C. J. Meskers, a. P. H. J. Schenning, and E. W. Meijer, Adv. Mater., 2003, 15, 1435-1438.

23. B. M. W. Langeveld-Voss, M. P. T. Christiaans, R. A. J. Janssen, and E. W. Meijer, Macromolecules, 1998, 31, 6702-6704.

24. M. M. Green and M. P. Reidy, J. Am. Chem. Soc., 1989, 111, 64526454.

25. M. M. Green, B. A. Garetz, B. Munoz, and H. Chang, J. Am. Chem. Soc., 1995, 117, 4181-4182.

55 26. M. Verswyvel, F. Monnaie, and G. Koeckelberghs, Macromolecules, 2011, 44, 9489-9498.

27. B. M. W. Langeveld-Voss, R. J. M. Waterval, R. A. J. Janssen, and E. W. Meijer, Macromolecules, 1999, 32, 227-230.

28. R. Fiesel and U. Scherf, Acta. Polym., 1998, 49, 445.

60 29. K. Van den Bergh, P. Willot, D. Cornelis, T. Verbiest, and G. Koeckelberghs, Macromolecules, 2011, 44, 728-735.

30. K. Van den Bergh, I. Cosemans, T. Verbiest, and G. Koeckelberghs, Macromolecules, 2010, 43, 3794-3800.

31. K. Van den Bergh, J. Huybrechts, T. Verbiest, and G. Koeckelberghs, Chem. Eur. J., 2008, 14, 9122-9125.

32. T. Mori, M. Kyotani, and K. Akagi, Macromolecules, 2010, 43, 8363-8372.

33. K. Akagi, Chem. Rev., 2009, 109, 5354-5401.

34. F. J. M. Hoeben, P. Jonkheijm, E. W. Meijer, and A. P. H. J.

70 Schenning, Chem. Rev., 2005, 105, 1491-546.
35. D. T. McQuade, A. E. Pullen, and T. M. Swager, Chem. Rev., 2000, 100, 2537-2574

36. E. Yashima, H. Goto, and Y. Okamoto, Macromolecules, 1999, 32, 7942-7945.

75 37. A. El-Ghayoury, A. P. H. J. Schenning, and E. W. Meijer, J. Polym. Sci., Part A: Polym. Chem., 2002, 40, 4020-4023.

38. M. Goh, T. Matsushita, M. Kyotani, and K. Akagi, Macromolecules, 2007, 40, 4762-4771.

39. M. J. Goh, M. Kyotani, and K. Akagi, Curr. Appl. Phys., 2006, 6, 948-951.

40. K. Araya, A. Mukoh, T. Narahara, and H. Shirakawa, Synth Met, 1986, 14, 199-206.

41. K. Akagi, G. Piao, S. Kaneko, K. Sakamaki, H. Shirakawa, and M. Kyotani, Science, 1998, 282, 1683-1686.

85 42. G. Piao, K. Akagi, H. Shirakawa, and M. Kyotani, Curr. Appl. Phys., $2001,1,121-123$

43. K. Akagi and T. Mori, Chem. Rec., 2008, 8, 395-406.

44. T. Mori, M. Kyotani, and K. Akagi, Chem. Sci., 2011, 2, 1389-1395.

45. K. Akagi, I. Higuchi, G. Piao, H. Shirakawa, and M. Kyotani, Mol.

90 Cryst. Liq. Cryst., 1999, 332, 463-470.

46. H. Goto and K. Akagi, Chem. Mater., 2006, 18, 255-262.

47. H. Goto and K. Akagi, Macromol. Rapid Commun., 2004, 25, 14821486.

48. H. Goto, N. Nomura, and K. Akagi, J. Polym. Sci., Part A: Polym. Chem., 2005, 43, 4298-4302.

49. H. Goto and K. Akagi, Macromolecules, 2005, 38, 1091-1098

50. H. Goto and K. Akagi, J. Polym. Sci., Part A: Polym. Chem., 2006, 44, 1042-1047.

51. H. Goto, T. Miyazawa, K. Tomishige, K. Kunimori, R. H. L. Kiebooms, Y. Akiyama, and K. Akagi, J. Appl. Polym. Sci., 2008, 107, 438-443.

52. F. Togashi, R. Ohta, and H. Goto, Tetrahedron Lett., 2007, 48, 2559 2562.

53. H. Goto, Phys. Rev. Lett., 2007, 98, 253901-1-253901-4.

105 54. Y. S. Jeong and K. Akagi, Macromolecules, 2011, 44, 2418-2426.

55. H. Goto, Macromolecules, 2007, 40, 1377-1385.

56. H. Goto and K. Akagi, Angew. Chem. Int. Ed., 2005, 44, 4322-4328.

57. S. Matsushita and K. Akagi, Isr. J. Chem., 2011, 51, 1075-1095.

58. H. Guo, C. M. Knobler, and R. B. Kaner, Synth. Met., 1999, 101, 441047.

59. C. Boonchu, L. A. P. Kane-Maguire, and G. G. Wallace, Synth. Met., 2003, 135-136, 241-242.

60. H. Goto, Polymer, 2008, 49, 3619-3624.

61. H. Yoneyama, A. Tsujimoto, and H. Goto, Macromolecules, 2007, 115 40, 5279-5283.

62. E. V. Strounina, L. A. P. Kane-Maguire, and G. G. Wallace, Polymer, 2006, 47, 8088-8094.

63. I. D. Norris, L. A. P. Kane-Maguire, and G. G. Wallace, Macromolecules, 2000, 33, 3237-3243.

120 64. E. V. Strounina, L. A. P. Kane-Maguire, and G. G. Wallace, Synth Met., 1999, 106, 129-137.

65. I. S. Vasil'eva, O. V. Morozova, G. P. Shumakovich, S. V. Shleev, I. Y. Sakharov, and a. I. Yaropolov, Synth. Met., 2007, 157, 684-689.

66. A. V. Caramyshev, V. M. Lobachov, D. V. Selivanov, E. V. Sheval, A. K. Vorobiev, O. N. Katasova, V. Y. Polyakov, A. a Makarov, and I. Y. Sakharov, Biomacromolecules, 2007, 8, 2549-2555.

67. Y. Kong, J. Ni, W. Wang, and Z. Chen, Electrochim. Acta, 2011, 56, 4070-4074.

68. M. R. Majidi, L. A. P. Kane-Maguire, and G. G. Wallace, Polymer, 1996, 37, 359-362.

69. M. R. Majidi, S. A. Ashraf, L. A. P. Kane-Maguire, I. D. Noms, and G. G. Wallace, Synth. Met., 1997, 84, 115-116.

70. S. A. Ashraf, L. A. P. Kane-Maguire, M. R. Majidi, S. G. Pyne, and G. G. Wallace, Polymer, 1997, 38, 2627-2631.

135 71. R. Dubey, D. Dutta, T. C. Shami, and K. U. B. Rao, Chirality, 2011, 23, 320-325.

72. J. Huang, V. M. Egan, H. Guo, J.-Y. Yoon, a. L. Briseno, I. E. Rauda, R. L. Garrell, C. M. Knobler, F. Zhou, and R. B. Kaner, Adv. Mater., 2003, 15, 1158-1161. 
73. J. Chen, B. Winther-Jensen, Y. Pornputtkul, K. West, L. A. P. KaneMaguire, and G. G. Wallace, Electrochem. Solid. St., 2006, 9, C9C11.

74. V. Egan, R. Bernstein, L. Hohmann, T. Tran, and R. B. Kaner, Chem. Commun., 2001, 801-802.

75. A. G. MacDiarmid, Synth. Met., 1997, 84, 27-34.

76. M. R. Majidi, L. A. P. Kane-Maguire, and G. G. Wallace, Polymer, 1995, 36, 3597-3599.

77. E. E. Havinga, M. M. Bouman, E. W. Meijer, A. Pomp, and M. M. J. Simenon, Synth. Met., 1994, 66, 93-97.

78. X. Yin, J. Ding, S. Zhang, and J. Kong, Biosens Bioelectron, 2006, 21, 2184-2187.

79. E. M. Sheridan and C. B. Breslin, Electroanal., 2005, 17, 532-537.

80. Y. Yang and M. Wan, J. Mater. Chem., 2002, 12, 897-901.

15 81. I. Basozabal, A. Gómez-Caballero, N. Unceta, M. Aranzazu Goicolea, and R. J. Barrio, Electrochim. Acta, 2011, 58, 729-735.

82. W. Li and H.-L. Wang, J. Am. Chem. Soc., 2004, 126, 2278-2279.

83. L. A. P. Kane-Maguire, A. G. MacDiarmid, I. D. Norris, G. G. Wallace, and W. Zheng, Synth. Met., 1999, 106, 171-176.

20 84. M. R. Majidi, L. A. P. Kane-Maguire, and G. G. Wallace, Aust. J. Chem., 1998, 51, 23-30.

85. I. D. Norris, L. A. P. Kane-Maguire, and G. G. Wallace, Macromolecules, 1998, 31, 6529-6533.

86. M. R. Majidi, L. A. P. Kane-Maguire, and G. G. Wallace, Polymer, 1994, 35, 3113-3115.

87. R. B. Kaner, Synth. Met., 2001, 125, 65-71.

88. K. Schügerl, R. Ulber, and T. Scheper, Trac-Trend. Anal. Chem., 1996, 15, 56-62.

89. E. Yashima and K. Maeda, Macromolecules, 2008, 41, 3-12.

30 90. E. Yashima, T. Matsushima, and Y. Okamoto, J. Am. Chem. Soc., 1997, 119, 6345-6359.

91. R. L. Elsenbaumer, H. Eckhardt, Z. Iqbal, J. Toth, and R. H. Baughman, Mol. Cryst. Liq. Cryst., 1985, 118, 111-116.

92. Y. Zhou, B. Yu, and G. Zhu, Polymer, 1997, 38, 5493-5495.

35 93. B. Deore, Z. Chen, and T. Nagaoka, Anal. Chem., 2000, 72, 39893994.

94. J. Huang, Z. Wei, and J. Chen, Sensor. Actuat. B-chem., 2008, 134, 573-578.

95. V. Syritski, J. Reut, A. Menaker, R. E. Gyurcsányi, and A. Öpik,

40 Electrochim. Acta, 2008, 53, 2729-2736.

96. E. Yashima, T. Matsushima, and Y. Okamoto, J. Am. Chem. Soc., 1995, 117, 11596-11597.

97. E. Yashima, K. Maeda, and Y. Okamoto, Nature, 1999, 399, 449451.

45 98. E. Yashima, K. Maeda, and T. Yamanaka, J. Am. Chem. Soc., 2000, 122, 7813-7814.

99. M. A. Saito, K. Maeda, H. Onouchi, and E. Yashima, Macromolecules, 2000, 33, 4616-4618.

100.H. Onouchi, K. Maeda, and E. Yashima, J. Am. Chem. Soc., 2001, 123, 7441-7442.

101.K. Maeda, H. Mochizuki, K. Osato, and E. Yashima, Macromolecules, 2011, 44, 3217-3226.

102.Y. Ashida, T. Sato, K. Morino, K. Maeda, Y. Okamoto, and E. Yashima, Macromolecules, 2003, 36, 3345-3350.

55 103.K. Maeda, K. Morino, Y. Okamoto, T. Sato, and E. Yashima, J. Am. Chem. Soc., 2004, 126, 4329-4342.

104.H. Onouchi, D. Kashiwagi, K. Hayashi, K. Maeda, and E. Yashima, Macromolecules, 2004, 37, 5495-5503.

105.H. Onouchi, T. Hasegawa, D. Kashiwagi, H. Ishiguro, K. Maeda, and

60 E. Yashima, J. Polym. Sci., Part A: Polym. Chem., 2006, 44, 50395048.

106.H. Onouchi, T. Hasegawa, D. Kashiwagi, H. Ishiguro, K. Maeda, and E. Yashima, Macromolecules, 2005, 38, 8625-8633.

107.T. Hasegawa, K. Maeda, H. Ishiguro, and E. Yashima, Polym. J., 2006, 38, 912-919.

108.M. Morimoto, K. Tamura, K. Nagai, and E. Yashima, J. Polym. Sci., Part A: Polym. Chem., 2010, 48, 1383-1390.

109.K. Maeda, K. Kuroyanagi, S.-ichiro Sakurai, T. Yamanaka, and E. Yashima, Macromolecules, 2011, 44, 2457-2464.
70 110.T. Nishimura, K. Tsuchiya, S. Ohsawa, K. Maeda, E. Yashima, Y. Nakamura, and J. Nishimura, J. Am. Chem. Soc., 2004, 126, 1171111717.

111.S. Vandeleene, M. Verswyvel, T. Verbiest, and G. Koeckelberghs, Macromolecules, 2010, 43, 7412-7423.

75 112.E. Yashima, Y. Maeda, and Y. Okamoto, Chem. Lett., 1996, 955-956.

113.E. Yashima, Y. Maeda, T. Matsushima, and Y. Okamato, Chirality, 1997, 9, 593-600

114.E. Yashima, Y. Maeda, and Y. Okamoto, J. Am. Chem. Soc., 1998, 120, $8895-8896$.

80 115.K. Maeda, S. Okada, E. Yashima, and Y. Okamoto, J. Polym. Sci., Part A: Polym. Chem., 2001, 39, 3180-3189.

116.K. Nagai, K. Maeda, Y. Takeyama, K. Sakajiri, and E. Yashima, Macromolecules, 2005, 38, 5444-5451.

117.E. Yashima, T. Nimura, T. Matsushima, and Y. Okamoto, J. Am. Chem. Soc., 1996, 118, 9800-9801.

118.H. Kawamura, K. Maeda, Y. Okamoto, and E. Yashima, Chem. Lett., 2001, 58-59.

119.R. Nonokawa and E. Yashima, J. Polym. Sci., Part A: Polym. Chem., 2003, 41, 1004-1013.

90 120.R. Nonokawa and E. Yashima, J. Am. Chem. Soc., 2003, 125, 12781283.

121.R. Nonokawa, M. Oobo, and E. Yashima, Macromolecules, 2003, 36, 6599-6606.

122.T. Nishimura, S. Ohsawa, K. Maeda, and E. Yashima, Chem. Commun., 2004, 646-647.

123.K. P. R. Nilsson, J. Rydberg, L. Baltzer, and O. Inganäs, P. Natl. Acad. Sci. USA, 2004, 101, 11197-11202.

124.Z. Yao, C. Li, and G. Shi, Langmuir, 2008, 24, 12829-12835.

125.C. Li, M. Numata, A.-H. Bae, K. Sakurai, and S. Shinkai, J. Am. 100 Chem. Soc., 2005, 127, 4548-4549.

126.C. Li, M. Numata, M. Takeuchi, and S. Shinkai, Angew. Chem. Int. Ed., 2005, 117, 6529-6532.

127.C. Li, M. Numata, M. Takeuchi, and S. Shinkai, Chem.-Asian. J., 2006, 1, 95-101.

105 128.H.-A. Ho, M. Boissinot, M. G. Bergeron, G. Corbeil, K. Doré, D. Boudreau, and M. Leclerc, Angew. Chem. Int. Ed., 2002, 41, 15481551.

129.H. A. Ho, K. Doré, M. Boissinot, M. G. Bergeron, R. M. Tanguay, D. Boudreau, and M. Leclerc, J. Am. Chem. Soc., 2005, 127, 1267312676.

130.K. Doré, S. Dubus, H.-A. Ho, I. Lévesque, M. Brunette, G. Corbeil, M. Boissinot, G. Boivin, M. G. Bergeron, D. Boudreau, and M. Leclerc, J. Am. Chem. Soc., 2004, 126, 4240-4244.

131.H.-A. Ho, M. Béra-Abérem, and M. Leclerc, Chem. Eur. J., 2005, 11, $1718-1724$

132.P. Ewbank, G. Nuding, H. Suenaga, R. D. McCullough, and S. Shinkai, Tetrahedron Lett., 2001, 42, 155-157.

133.B. Liu and G. C. Bazan, J. Am. Chem. Soc., 2004, 126, 1942-1943. 